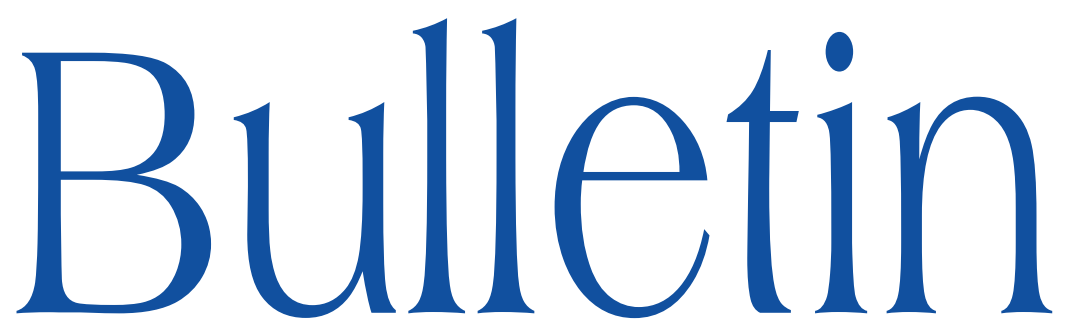

de la SOCIÉTÉ MATHÉMATIQUE DE FRANCE

\title{
MINIMAL SYSTEMS AND DISTRIBUTIONALLY SCRAMBLED SETS
}

Piotr Oprocha

Tome 140 Fascicule 3

2012 
Bull. Soc. math. France

140 (3), 2012, p. 401-439

\title{
MINIMAL SYSTEMS AND DISTRIBUTIONALLY SCRAMBLED SETS
}

\author{
BY PIOTR OPROCHA
}

\begin{abstract}
In this paper we investigate numerous constructions of minimal systems from the point of view of $\left(\mathscr{F}_{1}, \mathscr{F}_{2}\right)$-chaos (but most of our results concern the particular cases of distributional chaos of type 1 and 2). We consider standard classes of systems, such as Toeplitz flows, Grillenberger $K$-systems or Blanchard-Kwiatkowski extensions of the Chacón flow, proving that all of them are DC2. An example of DC1 minimal system with positive topological entropy is also introduced. The above mentioned results answer a few open problems known from the literature.
\end{abstract}

\section{Introduction}

The notion of distributional chaos was introduced by Schweizer and Smital in 1994 in [29] as a property equivalent to positive topological entropy for maps acting on the unit interval (it extends the notion of pair chaotic in the sense of Li and Yorke, which was know to be not sufficiently strong to imply positive topological entropy). Presently, we have at least three different definitions of distributionally chaotic pair [5] and it was also observed that uniform constant

Texte reçu le $1^{\text {er }}$ mars 2010, révisé le 2 mars 2011, accepté le 9 mai 2011.

Piotr Oprocha, Faculty of Applied Mathematics, AGH University of Science and Technology, al. Mickiewicza 30, 30-059 Kraków, Poland and Institute of Mathematics, Polish Academy of Sciences, ul. Śniadeckich 8, 00-956 Warszawa, Poland E-mail : oprocha@agh.edu.pl 2010 Mathematics Subject Classification. - 37B05; 37B40, 37B10.

Key words and phrases. - Chaotic pair, scrambled set, Mycielski set, distributional chaos, Li-Yorke chaos, filter. 
of separation of orbits may be important when dealing with this kind of chaos [23]. Recently, Xiong and Tan used in [31] families of subset of integers to define chaotic maps, obtaining that way an interesting and general definition. We adopt this approach here (all the necessary definitions are postponed to the next section).

In [29] the name distributional chaos do not appear explicitly, however it is proved there (in different terminology) that on the unit interval, one DC3 pair is enough to the existence of distributionally $\varepsilon$-scrambled set and both these properties are equivalent to positive topological entropy. It is also interesting that chaos means in [29] the existence of uncountable set whose any two distinct elements form a DC2 pair (so a condition somewhere in the middle between the above two properties). In general setting (i.e. beyond dimension one) there is no more such equivalence, that is, there are systems with positive topological entropy and no DC1 pairs [27] (even minimal ones [4]) as well as systems with DC1 pairs but entropy zero [22].

There are only a few general tools detecting distributionally scrambled sets (e.g. see [30, 3, 25]) however dynamics of systems fulfilling assumptions of these results is highly non-minimal. In the case of minimal maps some methods of construction have been developed, however they have either entropy zero (e.g. see $[22,23,31]$ ) or do not contain DC1 pairs (e.g. see [4]), while containing plenty of DC2 pairs. In fact, the most challenging conjecture related to distributional chaos (probably first stated by Smítal and then repeated by others, including the author himself) is that every system with positive entropy must contain a DC2 pair.

The main aim of this article is to examine various constructions of minimal systems with positive topological entropy (e.g. Toeplitz flows, extensions of Chacón flow, minimal K-systems, etc.) from the point of view of distributional chaos, or more generally $\left(\mathscr{F}_{1}, \mathscr{F}_{2}\right)$-chaos, where $\mathscr{F}_{1}, \mathscr{F}_{2}$ are upward hereditary sets of subsets of $\mathbb{N}$ (so-called Furstenberg families). That way we provide many methods of construction of minimal dynamical systems having uncountably many distributionally chaotic pairs (or not having them at all), filling a gap existing in the literature of the topic and answering a few open problems stated before (e.g. these stated by Balibrea and Smítal in [4]). Especially, two constructions contained in the paper can be of interest: a minimal system with positive entropy and DC1 pairs (see Theorem 9.3) and minimal system with positive entropy but without DC1 pairs nor regularly recurrent points (see Theorem 6.1). Second of this examples follows form a general fact that almost 1-1 extensions of minimal distal systems never have DC1 pairs (see Corollary 4.2). We also prove that every minimal u.p.e. system has plenty of DC2 pairs (see Theorem 7.6), which provides a partial answer (in a very restricted case) to the general conjecture on entropy and DC2 mentioned before.

TOME $140-2012-\mathrm{N}^{\mathrm{O}} 3$ 


\section{Preliminaries}

2.1. Basic notation. - In this paper $X$ is always assumed to be a compact metric space with a metric $d$, and $f: X \rightarrow X$ to be continuous. The set of all such maps is denoted $C(X)$. Open balls are denoted by $B(x, \varepsilon):=$ $\{y \in X: d(x, y)<\varepsilon\}$. The same notation is used for every nonempty set $A \subset$ $X$, that is $B(A, \varepsilon):=\bigcup_{x \in A} B(x, \varepsilon)$.

If $\left(X, d_{1}\right),\left(Y, d_{2}\right)$ are metric spaces, we always endow $X \times Y$ with the maximum metric $\rho((x, y),(p, q))=\max \left\{d_{1}(x, p), d_{2}(y, q)\right\}$. The diagonal in the product $X \times X$ is denoted $\Delta:=\{(x, x): x \in X\}$ and $\Delta_{\varepsilon}:=B(\Delta, \varepsilon)$ for any $\varepsilon>0$. By a perfect set we mean a nonempty compact set without isolated points and by a Cantor set we mean a perfect and totally disconnected set. If a set $A$ contains a countable intersection of open and dense subsets of $X$, then we say that $A$ is residual in $X$.

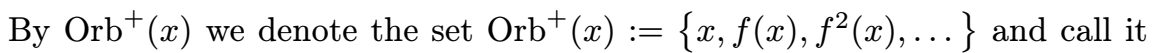
the (positive) orbit of a point $x$. If $f$ is invertible, then we define orbit of $x$ by $\operatorname{Orb}(x):=\left\{f^{i}(x): i \in \mathbb{Z}\right\}$. A point $y \in X$ is an $\omega$-limit point of a point $x$ if it is an accumulation point of the sequence $x, f(x), f^{2}(x), \ldots$ The set of all $\omega$-limit points of $x$ is said to be the $\omega$-limit set of $x$ or positive limit set of $x$ and is denoted $L^{+}(x, f)$; we reserve symbol $\omega$ to denote another property. We say that a point $x$ is periodic if $f^{n}(x)=x$ for some $n \geq 1$ and recurrent if $x \in L^{+}(x, f)$. Every set $M$ which is nonempty, closed, invariant (i.e. $f(M) \subset M$ ) and has no proper subset with these three properties is said to be a minimal set. If $X$ is the minimal set for $f$ then we say that $f$ is a minimal system. Elements of minimal system are usually said to be minimal points.

Points $x, y \in X$ are proximal, if $\lim _{\inf _{n \rightarrow \infty}} d\left(f^{n}(x), f^{n}(y)\right)=0$. We say that a point $x$ is distal, if it is not proximal to any point $y \in L^{+}(x, f) \backslash\{x\}$. We say that a nonempty set $A$ is synchronously proximal if $\liminf \inf _{n \rightarrow \infty} \operatorname{diam} f^{n}(A)=$ 0 . It is known that every point is proximal to some minimal point [11] (this statement is nontrivial when given point is not minimal), so distal points are always minimal. We say that $f$ is distal if all of its points are distal (by the above, such a system is always a disjoint sum of minimal systems).

Let $X$ and $Y$ be compact metric spaces and let $f \in C(X), g \in C(Y)$. If there is a continuous onto map $\phi: X \rightarrow Y$ with $\phi \circ f=g \circ \phi$, we say that $f$ and $g$ are semiconjugate (by $\phi$ ). The map $\phi$ is said to be a semiconjugacy (between $f$ and $g$ ) or a factor map, the map $g$ is said to be a factor of $f$ and the map $f$ is said to be an extension of $g$.

2.2. Families and filters. - Here we recall basic facts related to families and filters. Our notation follows [2] together with some concepts from [31]. 
$A$ (Furstenberg) family $\mathscr{F}$ is a collection of subsets of $\mathbb{N}$ which is upwards hereditary, that is

$$
F_{1} \in \mathscr{F} \text { and } F_{1} \subset F_{2} \quad \Longrightarrow \quad F_{2} \in \mathscr{F} \text {. }
$$

A family is proper if $\mathbb{N} \in \mathscr{F}$ and $\varnothing \notin \mathscr{F}$. Recall that a set $A \subset \mathbb{N}$ is thick if for every $n>0$ there is $i$ such that $\{i, i+1, \ldots, i+n\} \subset A$. We denote by $\mathscr{B}$ the family of infinite subsets of $\mathbb{N}$ and by $\tau \mathscr{B}$ the family of all thick subsets of $\mathbb{N}$.

For every family $\mathscr{F}$ we define its dual family by $k \mathscr{F}:=\{F \subset \mathbb{N}: \mathbb{N} \backslash F \notin \mathscr{F}\}$. Elements of the dual family $k \tau \mathscr{B}$ are said to be syndetic sets.

If $\mathscr{F}_{1}$ and $\mathscr{F}_{2}$ are families then we define

$$
\mathscr{F}_{1} \cdot \mathscr{F}_{2}:=\left\{F_{1} \cap F_{2}: F_{1} \in \mathscr{F}_{1}, F_{2} \in \mathscr{F}_{2}\right\} .
$$

Note that $\mathscr{F}_{1} \cup \mathscr{F}_{2} \subset \mathscr{F}_{1} \cdot \mathscr{F}_{2}$ for any two proper families $\mathscr{F}_{1}, \mathscr{F}_{2}$. We say that families $\mathscr{F}_{1}$ and $\mathscr{F}_{2}$ meet when $\mathscr{F}_{1} \cdot \mathscr{F}_{2}$ is proper. Let $\mathscr{P}(\mathbb{N})$ denote the power set of $\mathbb{N}$. For $\mathscr{A} \subset \mathscr{P}(\mathbb{N})$ we define the family generated by $\mathscr{A}$ as

$$
[\mathscr{A}]:=\{F \subset \mathbb{N}: A \subset F \text { for some } A \in \mathscr{A}\}
$$

For the case $\mathscr{A}=\{A\}$, where $A \subset \mathbb{N}$, we simply write $[A]$ instead of $[\mathscr{A}]$. For any infinite set $Q \in \mathscr{B}$ we define $\langle Q\rangle:=[T(Q)]$ where $T(Q)$ is the set of tails of $Q$, i.e. $T(Q)=\{Q \cap[n,+\infty): n \in \mathbb{N}\}$.

A filter $\mathscr{F}$ is a proper family such that $\mathscr{F} \cdot \mathscr{F}=\mathscr{F}$. Let $\mathscr{A} \subset \mathscr{P}(\mathbb{N})$ and denote $\mathscr{A}^{\cap}:=\left\{A_{1} \cap \cdots \cap A_{n}: A_{i} \in \mathscr{A}, n>0\right\}$. If $\varnothing \notin \mathscr{A}^{\cap}$ then [ $\left.\mathscr{A}^{\cap}\right]$ is a filter. In that case we say that $\mathscr{A}$ generates a filter and call $\left[\mathscr{A}^{\cap}\right]$ the filter generated by $\mathscr{A}$.

Note that, if $Q \in \mathscr{B}$ then $[Q]$ and $\langle Q\rangle$ are filters generated by $\{Q\}$ and $T(Q)$ respectively, since $\{Q\}^{\cap}=\{Q\}$ and $T(Q)^{\cap}=T(Q)$.

Given $A \subset X$ and $x \in X$ we write $N(x, A, f)=\left\{n: f^{n}(x) \in A\right\}$. If $A, B$ are sets then $N(A, B, f)=\left\{n: f^{-n}(B) \cap A \neq \varnothing\right\}$. We say that a point $x$ is uniformly recurrent if the set $N(x, U, f)$ is syndetic for every open set $U \ni x$. It is known that every element of a minimal set is uniformly recurrent, and $L^{+}(x, f)$ is a minimal set for every uniformly recurrent point (this was first proved by Birkhoff), in particular minimal or uniformly recurrent points define the same property in different language.

Let $P=\left\{p_{1}<p_{2}<\cdots\right\} \in \mathscr{B}$. Define

$$
\mathscr{D}^{*}(P):=\limsup _{n \rightarrow \infty} \frac{\#(P \cap\{1, \ldots, n\})}{n} \quad, \quad \mathscr{D}_{*}(P):=\liminf _{n \rightarrow \infty} \frac{\#(P \cap\{1, \ldots, n\})}{n} .
$$

We say that $\mathscr{D}^{*}(P)$ and $\mathscr{D}_{*}(P)$ are the upper density and the lower density of $P$ respectively. If $\mathscr{D}^{*}(P)=\mathscr{D}_{*}(P)$ then we denote $\mathscr{D}(P):=\mathscr{D}^{*}(P)$ and call this number the density of $P$. 
For any $t>0$ we define the following (proper) family

$$
\mathscr{M}(t):=\left\{P \in \mathscr{B}: \mathscr{D}^{*}(P) \geq t\right\} .
$$

The above mentioned families will be the main ingredient of most of the definitions appearing in the present paper.

\subsection{Scrambled sets}

Definition 2.1. - We say that an uncountable set $S \subset X$ is $\varepsilon$-scrambled if

$$
\liminf _{n \rightarrow \infty} d\left(f^{n}(x), f^{n}(y)\right)=0 \text { and } \limsup _{n \rightarrow \infty} d\left(f^{n}(x), f^{n}(y)\right)>\varepsilon
$$

for any distinct $x, y \in S$ and some $\varepsilon>0$. If the above conditions holds only with $\varepsilon=0$ then we say that $S$ is a scrambled set.

If there is a scrambled (or $\varepsilon$-scrambled set) for $f$ then we say that $f$ is chaotic (resp. $\varepsilon$-chaotic) in the sense of Li and Yorke.

The above definition originated from [21] and was later extended by numerous authors. Below we present a few such extensions which will be used in a further parts of the article.

For any positive integer $n$, points $x, y \in X$ and $t>0$, let

$$
\begin{aligned}
\Phi_{x y}^{(n)}(t) & :=\frac{1}{n} \#\left\{i: d\left(f^{i}(x), f^{i}(y)\right)<t \quad, 0 \leq i<n\right\}, \\
\Phi_{x y}(t) & :=\liminf _{n \rightarrow \infty} \Phi_{x y}^{(n)}(t)=\mathscr{D}_{*}\left(\left\{i: d\left(f^{i}(x), f^{i}(y)\right)<t\right\}\right), \\
\Phi_{x y}^{*}(t) & :=\limsup _{n \rightarrow \infty} \Phi_{x y}^{(n)}(t)=\mathscr{D}^{*}\left(\left\{i: d\left(f^{i}(x), f^{i}(y)\right)<t\right\}\right) .
\end{aligned}
$$

Definition 2.2. - Let $\varepsilon>0$. A pair $(x, y) \in X \times X$ is distributionally $\varepsilon$-scrambled if

$$
\Phi_{x y}(\varepsilon)=0 \quad \text { and } \quad \Phi_{x y}^{*}(t)=1 \quad \text { for all } t>0 .
$$

If a pair is distributionally $\varepsilon$-scrambled for some $\varepsilon>0$ then we simply call it distributionally scrambled. The sets of all distributionally $\varepsilon$-scrambled and distributionally scrambled pairs are denoted $\mathrm{DC}_{\varepsilon}(f)$ and $\mathrm{DC}(f)$ respectively.

We say that a set $S \subset X$ is distributionally $\varepsilon$-scrambled (resp. distributionally scrambled) if $S$ is uncountable and $S \times S \subset \mathrm{DC}_{\varepsilon}(f) \cup \Delta$ (resp. $S \times S \subset \mathrm{DC}(f) \cup \Delta)$.

The concept of distributional chaos originated from [29] and presently there are at least three versions of distributionally chaotic pair in the literature [5]. Furthermore, DC1 is completely independent of the value of topological entropy in general (there are examples of maps with entropy zero but DC1 [22] and viceversa [27]). More extensive introduction to the topic of distributional chaos can be found in [28]. 
Definition 2.3. - If there is an uncountable $S$ such that for every distinct $x, y \in S$ :

(DC1) $\Phi_{x y}^{*}(t)=1$ for all $t>0$ and $\Phi_{x y}(s)=0$ for some $s>0$,

(DC2) $\Phi_{x y}^{*}(t)=1$ for all $t>0$ and $\Phi_{x y}(s)<1$ for some $s>0$,

(DC3) $\Phi_{x y}^{*}(t)>\Phi_{x y}(t)$ for all $t \in J$, where $J$ is some nondegenerate interval,

then we say that $f$ is distributionally chaotic of type 1, 2 or 3 respectively (DC1, DC2 or DC3 for short).

Note that $f$ has a distributionally scrambled set if and only if it is DC1. The following three definitions were introduced in [31].

Definition 2.4. - Let $\mathscr{F}$ be a family and $A \subset X$ be nonempty. Then $x \in X$ is an $\mathscr{F}$-attaching point of $A$ if $N(x, A, f) \in \mathscr{F}$. We call the set

$$
\mathscr{F}(A, f):=\bigcup_{F \in \mathscr{F}} \bigcap_{i \in F} f^{-i}(A)
$$

the $\mathscr{F}$-attaching set of $\mathrm{A}$.

Note that $\mathscr{F}(A, f)=\{x: x$ is $\mathscr{F}$-attaching point of $A\}$.

Definition 2.5. - Let $\mathscr{F}$ be a family and $A \subset X$ be a nonempty set. We say that a point $x \in X$ is an $\mathscr{F}$-adherent point of $A$, if it is an $\mathscr{F}$-attaching point of $B(A, \varepsilon)$ for any $\varepsilon>0$.

The set of all $\mathscr{F}$-adherent points of $A$ is denoted

$$
\begin{aligned}
\mathscr{F}^{+}(A, f) & :=\{x: N(x, B(A, \varepsilon), f) \in \mathscr{F} \text { for any } \varepsilon>0\} \\
& =\bigcap_{\varepsilon>0} \mathscr{F}(B(A, \varepsilon), f) .
\end{aligned}
$$

Note that if $\delta<\varepsilon$ and $N(x, B(A, \delta), f) \in \mathscr{F}$ then also $N(x, B(A, \varepsilon), f) \in \mathscr{F}$ since $\mathscr{F}$ is a family. But then we can write

$$
\mathscr{F}^{+}(A, f)=\bigcap_{n>0} \mathscr{F}\left(B\left(A, \frac{1}{n}\right), f\right) .
$$

Definition 2.6. - Let $(X, d)$ be compact, let $f \in C(X)$ and let $\mathscr{F}_{1}, \mathscr{F}_{2}$ be families. We say that $f$ is $\left(\mathscr{F}_{1}, \mathscr{F}_{2}\right)$-chaotic, if there exists an uncountable set $K \subset X$ such that for any distinct points $x, y \in K$ the pair $(x, y)$ fulfils the condition

$$
(x, y) \in \mathscr{F}_{1}^{+}(\Delta, f \times f) \cap \mathscr{F}_{2}\left(X \times X \backslash \Delta_{\gamma}, f \times f\right)
$$

with some $\gamma=\gamma(x, y)>0$.

If there is one global $\varepsilon=\varepsilon(K)>0$ such that

$$
(x, y) \in \mathscr{F}_{1}^{+}(\Delta, f \times f) \cap \mathscr{F}_{2}\left(X \times X \backslash \Delta_{\varepsilon}, f \times f\right)
$$

TOME $140-2012-\mathrm{N}^{\mathrm{O}} 3$ 
for any distinct points $x, y \in K$, then we say that $f$ is $\left(\mathscr{F}_{1}, \mathscr{F}_{2}\right)-\varepsilon$-chaotic.

The uncountable set $K$ in the above definitions is said to be $\left(\mathscr{F}_{1}, \mathscr{F}_{2}\right)$-scrambled set and $\left(\mathscr{F}_{1}, \mathscr{F}_{2}\right)$ - $\varepsilon$-scrambled set respectively.

2.4. Symbolic dynamics. - Let $\mathscr{G}$ be a finite set (an alphabet) and denote by $\mathscr{C}^{*}$ the set of all finite words over $\mathscr{G}$. The set $\mathscr{C}^{*}$ with the concatenation of words is a free monoid with the minimal set of generators $\mathscr{C}$ (we assume that the empty word, denoted by $\lambda$ is in $\left.\mathscr{Q}^{*}\right)$. The set of nonempty words is denoted by $\mathscr{G}^{+}$. If $w \in \mathscr{G}^{+}$and $k>0$ then by $w^{k}=w w \cdots w$ we denote the $k$-times concatenation of $w$ with itself. If $w=a_{0} a_{1} \cdots a_{k-1} \in \mathscr{C}^{+}$then we write $w_{i}:=a_{i}$ or $w[i]:=a_{i}$; the number $|w|=k$ is said to be the length of $w$; by $|w|_{a}$ is denoted the number of occurrences of the letter $a$ in $w$.

If $x \in \mathscr{G}^{\mathbb{N}}$ and $i \leq j$ are integers then we denote $x_{[i, j]}=x_{i} x_{i+1} \ldots x_{j}$ and $x_{[i, j)}=x_{[i, j-1]}$. If $i>j$ then $x_{[i, j]}=\lambda$, where $\lambda$ is the empty word. If $x=y$ then we put $d(x, y)=0$ and $d(x, y)=2^{-k}$ otherwise, where $k \geq 0$ is the maximal number such that $x_{[0, k)}=y_{[0, k)}$. We also define the shift map $\sigma: \mathscr{G}^{\mathbb{N}} \rightarrow \mathscr{G}^{\mathbb{N}}$ by putting $\sigma(x)_{i}=x_{i+1}$ for all $i \in \mathbb{N}$. It can be easily verified that $\left(\mathscr{C}^{\mathbb{N}}, d\right)$ is a compact metric space and $\sigma$ is continuous. For any $w \in \mathscr{G}^{+}$we define its cylinder set $C[w]:=\left\{x \in \mathscr{G}^{\mathbb{N}} ; x_{[0, n)}=w\right\}$ where $n=|w|$. It is well know that cylinder sets form a basis of the topology of $\left(\mathscr{C}^{\mathbb{N}}, d\right)$.

Any closed set $X$ invariant under $\sigma$ (i.e. $\sigma(X) \subset X$ ) is said to be a shift or a subshift. For simplicity, we write $C_{X}[w]=C[w] \cap X$ where $X$ is a subshift of $\left(\mathscr{G}^{\mathbb{N}}, d\right)$. By $\mathscr{L}(X)$ we denote the language of subshift $X$, that is the set $\mathscr{L}(X):=\left\{x_{[0, k]}: x \in X, k \geq 0\right\}$. We write $\mathscr{L}_{n}(X):=\{w \in \mathscr{L}(X):|w|=n\}$ for every $n>0$.

Similarly, we can define two-sided subshifts of $\mathscr{Q}^{\mathbb{Z}}$ with the only difference that in the definition of metric $k$ is the maximal number such that $x_{(-k, k)}=$ $y_{(-k, k)}$. In that case all other definitions presented above are modified accordingly.

2.5. Odometers and Toeplitz flows. - The reader not familiar with Toeplitz flows is refereed to [9] or [20, Ch. 4]. Here we briefly recall main definitions used later.

Let $\mathbf{s}=\left\{s_{m}\right\}_{m=1}^{\infty}$ be a sequence of positive integers such that $s_{m}$ divides $s_{m+1}$. We call such a sequence a scale. If we endow the cyclic group $\mathbb{Z}_{n}$ with the discrete topology, and define $\pi_{m}: \mathbb{Z}_{s_{m+1}} \rightarrow \mathbb{Z}_{s_{m}}$ by $\pi_{m}(z)=z\left(\bmod s_{m}\right)$ then the inverse limit

$$
G_{\mathbf{s}}=\lim _{\longleftarrow}\left\{\mathbb{Z}_{s_{m}}, \pi_{m}\right\}=\left\{\left\{x_{n}\right\}_{n=1}^{\infty}: \pi_{m}\left(x_{m+1}\right)=x_{m}\right\}
$$

is well defined, compact subset of the countable Cartesian product of $\mathbb{Z}_{s_{m}}$ with the product topology. Denote $\mathbf{1}:=(1,1, \ldots)$. By the odometer on scale $\mathbf{s}$ we 
mean $G_{\mathbf{s}}$ together with the map $R_{\mathbf{s}}: G_{\mathbf{s}} \rightarrow G_{\mathbf{s}}$ defined by $R_{\mathbf{s}}(\mathbf{j})=\mathbf{j}+\mathbf{1}$, where the addition is coordinatewise, modulo $s_{m}$ on each coordinate $m$. We say that an odometer is nontrivial if $G_{\mathbf{s}}$ is infinite (hence it is a Cantor set).

Let $\pi: X \rightarrow Y$ be a factor map (between $f \in C(X)$ and $g \in C(Y)$ ), and let $Y_{1}$ denote the set of points $y \in Y$ whose fibers $\pi^{-1}(\{y\})$ are singletons. We say that $f$ is almost 1-1 extension of $g$ (via the factor map $\pi$ ) if $Y_{1}$ is residual in $Y$.

We say that a point $x \in X$ is regularly recurrent if for every open neighborhood $U$ of $x$ there is $l>0$ such that $N(x, U, f) \supset l \mathbb{N}$. Any regularly recurrent point of $\sigma$ in $\{0,1\}^{\mathbb{N}}$ is said to be Toeplitz sequence. Each subshift $X_{\omega}$ generated by a Toeplitz sequence $\omega$ is said to be a Toeplitz flow (i.e. $X_{\omega}=\overline{\mathrm{Orb}^{+}(\omega, \sigma)}$ ). Elements of a Toeplitz flow are said to be Toeplitz orbitals.

It is well known that a minimal system $f \in C(X)$ is an almost 1-1 extension of an odometer $G_{\mathbf{S}}$ if and only if it is the orbit closure of a regularly recurrent point (e.g. see [9, Theorem 5.1]). For a Toeplitz orbital $x$ and an integer $s>0$, we denote

$$
\operatorname{Per}_{s}(x)=\{n \in \mathbb{N}: x(n)=x(n+k s) \text { for every } k=1,2, \ldots\} .
$$

If $\omega$ is a Toeplitz sequence then $\mathbb{N}=\bigcup_{m} \operatorname{Per}_{m}(\omega)$. By an essential period of $\omega$ we mean any $s$ such that $\operatorname{Per}_{s}(\omega) \neq \varnothing$ and does not coincide with $\operatorname{Per}_{k}(\omega)$ for any $k<s$. A period structure of $\omega$ is any sequence $\mathbf{s}=\left\{s_{m}\right\}_{m=1}^{\infty}$ of essential periods such that each $s_{m}$ divides $s_{m+1}$ and $\mathbb{N}=\bigcup_{m} \operatorname{Per}_{s_{m}}(\omega)$. It is known that a periodic structure always exists, and $X_{\omega}$ is an almost 1-1 extension of the odometer $G_{\mathbf{s}}$. So we will always assume that a periodic structure is fixed together with the factor map $\pi_{\omega}: X_{\omega} \rightarrow G_{\mathbf{s}}$. If $x$ is a Toeplitz orbital, then it may happen that periodic parts do not cover whole $\mathbb{N}$, that is the aperiodic part of $x$

$$
\operatorname{Aper}(x):=\mathbb{N} \backslash \bigcup_{m=1}^{\infty} \operatorname{Per}_{s_{m}}(x)
$$

is nonempty.

2.6. Topological entropy and entropy pairs. - The topological entropy of a system was first defined by Adler, Konheim and McAndrew [1]. Let $\mathscr{C}_{X}$ be the set of all finite open covers of $X$. Given two covers $\mathcal{u}, v \in \mathscr{C}_{X}$ denote

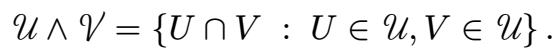

Let $r(\mathcal{U})$ denote the minimum among the cardinalities of subsets of $\mathcal{U} \in \mathscr{C}_{X}$ that cover $X$. The topological entropy of the cover $\mathcal{U} \in \mathscr{C}_{X}$ is defined as

$$
h_{\mathrm{top}}(f, \mathcal{U})=\lim _{n \rightarrow \infty} \frac{1}{n} \log r\left(\bigvee_{i=0}^{n-1} f^{-i}(\mathcal{U})\right) \text {. }
$$

TOME $140-2012-\mathrm{N}^{\mathrm{O}} 3$ 
where the existence of the limit and the second identity follow from the fact that $\left\{\log r\left(\bigvee_{i=0}^{n-1} f^{-i}(\mathcal{U})\right)\right\}_{n \in \mathbb{N}}$ is a sub-additive sequence. The topological entropy of $f$ is the number $h_{\text {top }}(f) \in[0,+\infty]$ defined by $h_{\text {top }}(f)=\sup _{\mathcal{U} \in \mathscr{C}_{X}} h_{\text {top }}(f, \mathcal{U})$.

Recall that a set $A$ is $(n, \varepsilon)$-separating if for every distinct $x, y \in A$ there is $0 \leq i \leq n$ such that $d\left(f^{i}(x), f^{i}(y)\right)>\varepsilon$. We will denote by $S_{A}(n, \varepsilon)$ the maximal number of elements in any $(n, \varepsilon)$-separating set contained in $A$. It is well known that on compact metric spaces:

$$
h_{\mathrm{top}}(f)=\lim _{\varepsilon \rightarrow 0^{+}} \limsup _{n \rightarrow \infty} \frac{1}{n} \log S_{X}(n, \varepsilon) .
$$

We say that a surjective $f \in C(X)$ has uniformly positive entropy (u.p.e.) if for each non-trivial (i.e. not containing $X$ ) open cover $\mathcal{U}$ consisting of two elements we have $h_{\text {top }}(f, \mathcal{U})>0$.

In all calculations related to entropy we assume that logarithms have base 2 , i.e. $\log (\cdot)$ always stands for $\log _{2}(\cdot)$.

\section{A family of minimal shifts (Xiong and Tan construction)}

In this section we extend the technique introduced in [31]. The first of all, we think that the class of minimal systems presented in [31] is interesting enough to deserve further investigation and additionally this class fits nicely in our research. Strictly speaking, for every $a \in[0,1)$ we will use Tan and Xiong ideas to construct a minimal shift $X_{a}$ over the alphabet $\{0,1\}$ such that for every $\varepsilon \in(0,1)$ the shift map $\sigma$ restricted to $X_{a}$ is $(\mathscr{M}(1), \mathscr{M}(a))$ - $\varepsilon$-chaotic but is not $(\mathscr{M}(1), \mathscr{M}(1))$-chaotic. In fact, we show even more, since there is a Cantor set $K \subset X_{a}$ and $Q \in \mathscr{M}(1)$ with the property that for every $n=1,2, \ldots$ there is $Q_{n} \in\langle Q\rangle$ such that

$$
K \times K \backslash \Delta \subset\left[Q_{n}\right]\left(\Delta_{1 / n}, \sigma \times \sigma\right) \cap \mathscr{M}(a)\left(X_{a} \times X_{a} \backslash \Delta_{\varepsilon}, \sigma \times \sigma\right) .
$$

In particular $K$ is synchronously proximal, and the set of iterations during which diam $K<\delta$ is of upper density 1 for any $\delta>0$. Furthermore, $X_{a}$ can be arbitrarily close to the whole space $\{0,1\}^{\mathbb{N}}$ in the sense of Hausdorff distance. As we said before, our construction generalizes ideas of [31]. Later, in further parts of the article, we will present another method of creation of examples with the above mentioned chaotic properties. That method will be less transparent (the construction will be obtained via an application of strong results in topological dynamics), however the dynamical properties of the constructed family will be also a little bit stronger (the topological entropy will be positive).

Now, we are ready to start our construction. Fix any $a \in[0,1)$ and $\theta \in(a, 1)$. Fix words $v_{1}, \ldots, v_{s}$ and let $u=v_{1} v_{2} \cdots v_{s}$. We add a few symbols 0 at the end of $u$ if necessary, obtaining $|u|>6$. 
First, we will construct inductively a family of words $\left\{A_{i}, \bar{A}_{i}\right\}_{i=1}^{\infty} \subset\{0,1\}^{+}$. The desired subshift will be obtained as the orbit closure of the infinite concatenation of some of these words.

Let $k_{1}>1$ be such that $3|u| / k_{1}<1-\theta$. Define

$$
A_{1}=0^{|u|+2} 1 u 11(01)^{k_{1}}, \quad \bar{A}_{1}=0^{|u|+2} 1 u 1(01)^{k_{1}} 1 .
$$

Observe, that $\left|A_{1}\right|=\left|\bar{A}_{1}\right|$ and $\eta(1)>\theta$ where

$$
\eta(1):=\frac{1}{\left|A_{1}\right|} \#\left\{0 \leq j<\left|A_{1}\right|: A_{1}(j) \neq \bar{A}_{1}(j)\right\}
$$

since by the definition

$$
\eta(1)=\frac{2 k_{1}+1}{2 k_{1}+2|u|+5} \geq 1-\frac{3|u|}{k_{1}}>\theta .
$$

Next, suppose that words $A_{i}, \bar{A}_{i} \in\{0,1\}^{+}$are defined such that $\left|A_{i}\right|=\left|\bar{A}_{i}\right|$, $A_{i} \neq \bar{A}_{i}$ and $\eta(i)>\theta$ for all $i=1, \ldots, n$, where

$$
\eta(i):=\frac{1}{\left|A_{i}\right|} \#\left\{0 \leq j<\left|A_{i}\right|: A_{i}(j) \neq \bar{A}_{i}(j)\right\} .
$$

There is $\alpha \in(0,1)$ such that $\eta(i)>\theta+\alpha$ for every $i=1, \ldots, n$. Fix a positive integer $k_{n+1}$ such that $2 / k_{n+1}<\alpha$. Let

$$
\mathscr{P}_{n}:=\left\{B_{1} \cdots B_{n}: B_{i} \in\left\{A_{i}, \bar{A}_{i}\right\}, 1 \leq i \leq n\right\} .
$$

For any $P \in \mathscr{P}_{n}, P=B_{1} \cdots B_{n}$ denote $\bar{P}=\bar{B}_{1} \cdots \bar{B}_{n}$, where $\overline{\bar{A}}_{i}=A_{i}$. Assume that elements of $\mathscr{P}_{n}$ are enumerated, let say $\mathscr{P}_{n}=\left\{P_{1}, \ldots, P_{s}\right\}$. Assume additionally that $\bar{P}_{1}=P_{2}$. Note that $s=2^{n}$ since $A_{i} \neq \overline{A_{i}}$ for every $i$. Define $A_{n+1}$ and $\bar{A}_{n+1}$ by

$$
\begin{aligned}
& A_{n+1}:=P_{1}\left(P_{1}\right)^{k_{n+1}}\left(P_{2}\right)^{k_{n+1}} \cdots\left(P_{s}\right)^{k_{n+1}} \\
& \bar{A}_{n+1}:=P_{1}\left(\bar{P}_{1}\right)^{k_{n+1}}\left(\bar{P}_{2}\right)^{k_{n+1}} \cdots\left(\bar{P}_{s}\right)^{k_{n+1}} .
\end{aligned}
$$

First note that $A_{n+1} \neq \bar{A}_{n+1}$ and $\left|A_{n+1}\right|=\left|\bar{A}_{n+1}\right|$. To proceed with induction, it remains to show that $\eta(n+1)>\theta$. To prove that, observe that $\left|P_{i}\right|=\left|P_{1}\right|$ for $i=1, \ldots, s$, and so

$$
\begin{aligned}
\eta(n+1) & \geq \frac{k_{n+1}}{\left|P_{1}\right|\left(s k_{n+1}+1\right)} \sum_{j=1}^{s} \#\left\{0 \leq i<\left|P_{1}\right|: P_{j}[i] \neq \bar{P}_{j}[i]\right\} \\
& \geq \frac{k_{n+1}}{\left|P_{1}\right|\left(s k_{n+1}+1\right)} \sum_{j=1}^{s} \sum_{i=1}^{n}\left|A_{i}\right| \eta(i) \\
& \geq \frac{k_{n+1}}{\left|P_{1}\right|\left(s k_{n+1}+1\right)} \sum_{j=1}^{s} \sum_{i=1}^{n}\left|A_{i}\right|(\theta+\alpha)
\end{aligned}
$$

TOME $140-2012-\mathrm{N}^{\circ} 3$ 


$$
\begin{aligned}
& \geq \frac{s k_{n+1}}{s k_{n+1}+1}(\theta+\alpha) \geq \theta+\alpha-\frac{2}{k_{n+1}} \\
& >\theta
\end{aligned}
$$

By the induction, a word $A_{i}$ with the properties specified above is well defined for $i=1,2, \ldots$.

Put $x_{a}:=A_{1} A_{2} A_{3} \cdots$ and define $X_{a}:=L^{+}\left(x_{a}, \sigma\right)$. Further in this section (see Lemma 3.3) we will show that $X_{a}$ is minimal for every parameter $a \in[0,1)$.

Lemma 3.1. - For every $n>0$ there is a sequence $\left\{i_{j}\right\}_{j=1}^{\infty} \subset\left\{1, \ldots, 2^{n}\right\}$ such that

$$
x_{a}=P_{i_{1}} P_{i_{2}} \cdots
$$

where $\mathscr{P}_{n}=\left\{P_{1}, \ldots, P_{2^{n}}\right\}$ is defined by (3.1).

Proof. — The proof is straightforward, thus left to the reader.

Lemma 3.2. - Fix any $n>0$ and $P, Q, R \in \mathscr{P}_{n}$. If there are $x, y \in\{0,1\}^{*}$ such that $P Q=x R y$ then $x=\lambda$ or $y=\lambda$.

Proof. - For $n=1$ the statement of the Lemma is obvious, since $0^{|u|+2}$ can be only a prefix of a word in $\mathscr{P}_{1}=\left\{A_{1}, \overline{A_{1}}\right\}$. Next assume that the statement holds for all $n=1, \ldots, m$ and fix any $P, Q, R \in \mathscr{P}_{m+1}$. There are $B_{1}, \ldots, B_{r}, C_{1}, \ldots, C_{r}, D_{1}, \ldots, D_{r} \in \mathscr{P}_{m}$ such that $P=B_{1} \cdots B_{r}, Q=$ $C_{1} \cdots C_{r}, R=D_{1} \cdots D_{r}$ where $r=1+2^{m} k_{m+1}$. Now assume that $P Q=x R y$ for some $x, y \in\{0,1\}^{*}$. If $x=\lambda$ or $x=P$ then we are done, so assume contrary that $|x| \in(0,|P|)$. By the assumptions of the induction, there is $s<r$ such that $x=B_{1} \cdots B_{s}$. Note that $B_{j}, D_{j} \notin\left\{P_{1}, P_{2}\right\}=\left\{\bar{P}_{1}, \bar{P}_{2}\right\}$ for $j>2 k_{m+1}+1$, while $C_{j}, D_{j} \in\left\{P_{1}, P_{2}\right\}$ for $j \in\left[1,2+2 k_{m+1}\right)$. If $s \in\left[1,2+2 k_{m+1}\right)$ then $D_{r}=C_{s} \in\left\{P_{1}, P_{2}\right\}$ and if $s \in\left[1+2 k_{m+1}, r-1\right]$ then $B_{s+1}=D_{1} \in\left\{P_{1}, P_{2}\right\}$. Both situations are impossible.

Lemma 3.3. - For every $a \in[0,1)$, the map $\sigma$ is minimal on $X_{a}$.

Proof. - First note that words $x=A_{1} \cdots A_{n}$ and $y=\bar{A}_{1} \cdots \bar{A}_{n}$ are contained in $\mathscr{P}_{n}$ and so both $x, y$ are subwords of $A_{n+1}$ and $\bar{A}_{n+1}$ (in both $A_{n+1}, \bar{A}_{n+1}$ all words from the set $\mathscr{P}_{n}$ have to appear, however in a different order). This implies that both $x, y$ are subwords of every word in $\mathscr{P}_{n+1}$. But then by Lemma 3.1 we obtain

$$
\left\{j: x_{a}[i+j]=w[i], 0 \leq i \leq|w|\right\} \in k \tau \mathscr{B}
$$

for any $w$ of the form $w=A_{1} A_{2} \cdots A_{n}$, which in other words means that $x_{a}$ is uniformly recurrent. This shows that $X_{a}$ is a minimal set for $\sigma$. 
Theorem 3.4. - For every $a \in[0,1)$ every proper family $\mathscr{F}$ and every $\gamma>0$

$$
\mathscr{F}^{+}(\Delta, \sigma \times \sigma) \cap \tau \mathscr{B}\left(X_{a} \times X_{a} \backslash \Delta_{\gamma}, \sigma \times \sigma\right)=\varnothing .
$$

In particular, the shift map $\sigma=\left.\sigma\right|_{X_{a}}$ is not $(\mathscr{M}(1), \mathscr{M}(1))$-chaotic.

Proof. - Suppose contrary to the statement of the theorem that there are $x, y \in X_{a}$ and $\gamma>0$ such that

$$
(x, y) \in \mathscr{F}^{+}(\Delta, \sigma \times \sigma) \cap \tau \mathscr{B}\left(X_{a} \times X_{a} \backslash \Delta_{\gamma}, \sigma \times \sigma\right) .
$$

We may assume that $x \neq y$, since obviously

$$
\Delta \cap \tau \mathscr{B}\left(X_{a} \times X_{a} \backslash \Delta_{\gamma}, \sigma \times \sigma\right)=\varnothing .
$$

There is $n>1$ such that $2^{-n}<\gamma$. By Lemma 3.1, without loss of generality we may assume that

$$
x=P_{i_{1}} P_{i_{2}} \cdots \quad, \quad y=v P_{k_{1}} P_{k_{2}} \cdots
$$

for some word $v \in\{0,1\}^{*}$ with $0 \leq|v|<\left|P_{1}\right|$ and some sequences $\left\{i_{j}\right\}_{j=1}^{\infty},\left\{k_{j}\right\}_{j=1}^{\infty} \subset\left\{1, \ldots, 2^{n}\right\}$, where $\mathscr{P}_{n}=\left\{P_{1}, \ldots, P_{2^{n}}\right\}$ is defined by (3.1).

Denote $m=|v|$. First assume that $m>0$. We claim that the set

$$
F=\left\{i: x_{i} \neq y_{i}\right\}
$$

is syndetic. Otherwise for $l=3\left|P_{1}\right|$ there is $i$ such that $x_{i+j}=y_{i+j}$ for $j=$ $0, \ldots, l$. By (3.2) there are $z, z^{\prime}, w, w^{\prime}$ and $P, Q, R \in \mathscr{P}_{n}$ such that $\left|z^{\prime}\right|=|z|+m$ and $z P Q w=z^{\prime} R w^{\prime}=x_{i} \cdots x_{i+k-1}$. We obtain a contradiction by Lemma 3.2. Indeed $F$ is syndetic. But then there is $\varepsilon>0$ such that

$$
N\left((x, y), \Delta_{\varepsilon}, \sigma \times \sigma\right) \subset\left\{i: d\left(\sigma^{i}(x), \sigma^{i}(y)\right)<2 \varepsilon\right\}=\varnothing .
$$

Since $\mathscr{F}$ is proper, $(x, y) \notin \mathscr{F}^{+}(\Delta, \sigma \times \sigma)$ which contradicts assumptions about the pair $(x, y)$. Then the only possibility is $m=0$. But there is a word $w \in \mathscr{P}_{n-1}$ such that $w$ is a prefix of every word in $\mathscr{P}_{n}$. Note that $|w| \geq(n-1)\left|A_{1}\right|>n$ and that the set

$$
E=\left\{i: x_{i+j}=y_{i+j}=w_{j} \text { for } 0 \leq j<|w|\right\}
$$

is syndetic, that is $E \in k \tau \mathscr{B}$. Furthermore, if $x_{i+j}=y_{i+j}$ for $0 \leq j<n$ then $d\left(\sigma^{i}(x), \sigma^{i}(y)\right)<\gamma$. This immediately implies that

$$
N\left((x, y), X_{a} \times X_{a} \backslash \Delta_{\gamma}, \sigma \times \sigma\right) \subset \mathbb{N} \backslash E \notin \tau \mathscr{B} .
$$

and so $(x, y) \notin \tau \mathscr{B}\left(X \times X \backslash \Delta_{\gamma}, \sigma \times \sigma\right)$ which is a contradiction. 
Theorem 3.5. - For every $a \in[0,1)$ and every $\varepsilon<1 / 2$ there is a Cantor set $K \subset X_{a}$ and a sequence of sets $\left\{Q_{n}\right\}_{n=1}^{\infty} \in \mathscr{M}(1)$ such that $Q_{n+1} \subset Q_{n}$ and

$$
K \times K \backslash \Delta \subset\left[Q_{n}\right]\left(\Delta_{1 / n}, \sigma \times \sigma\right) \cap \mathscr{M}(a)\left(X_{a} \times X_{a} \backslash \Delta_{\varepsilon}, \sigma \times \sigma\right) .
$$

for every $n=1,2, \ldots$.

Proof. - Let $\Xi \subset\{0,1\}^{\mathbb{N}}$ be an uncountable set, such that if $\alpha, \beta \in \Xi$ are distinct, then

$$
\#\left\{i: \alpha_{i}=\beta_{i}\right\}=\infty \quad, \quad \#\left\{i: \alpha_{i} \neq \beta_{i}\right\}=\infty .
$$

There are numerous methods for constructing such sets; e.g. we may follow [26] and for every $x \in\{0,1\}^{\mathbb{N}}$ define $\alpha_{x} \in\{0,1\}^{\mathbb{N}}$ putting

$$
\alpha_{x}=x_{0} 0 x_{0} x_{1} 0 x_{0} x_{1} x_{2} 0 x_{0} \cdots \in\{0,1\}^{\mathbb{N}} .
$$

Now it is enough to define $\Xi=\left\{\alpha_{x}: x \in\{0,1\}^{\mathbb{N}}\right\}$. Next, for any $\alpha \in \Xi$ define $z^{\alpha}=B_{1}^{\alpha} B_{2}^{\alpha} B_{3}^{\alpha} \cdots$ where $B_{i}^{\alpha}=A_{i}$ if $\alpha_{i}=0$ and $B_{i}^{\alpha}=\bar{A}_{i}$ otherwise. We put

$$
K=\left\{z^{\alpha}: \alpha \in \Xi\right\} \text {. }
$$

Note that $K$ is a Cantor set since maps $x \mapsto \alpha_{x}$ and $\alpha \mapsto z^{\alpha}$ are continuous injections.

For any $n>0$ denote $l_{n}=\left|A_{1} \cdots A_{n}\right|$. Let $\xi=101^{2} 01^{3} 01^{4} 01^{5} 0 \cdots$ and observe that if $\xi_{i}=0$ then $\alpha_{i}=0$ for any $\alpha \in \Xi$. Define

$$
Q=\bigcup_{\left\{i: \xi_{i}=0\right\}}\left[l_{i}, l_{i+1}-i\right) \cap \mathbb{N} .
$$

Observe that if we fix any $m$ and next take $n>m$ then $z^{\alpha}[i+j]=A_{n+1}\left[i-l_{n}+j\right]$ for every $i \in Q \cap\left[l_{n}, l_{n+1}\right)$, every $j \in\{0, \ldots, m\}$ and every $\alpha \in \Xi$. Additionally, $\xi_{n}=0$ if and only if $Q \cap\left[l_{n}, l_{n+1}\right) \neq \varnothing$.

By (3.1), if $P \in \mathscr{P}_{n}$ then $|P|=l_{n}$, which implies that

$$
\left|A_{n+1}\right|=\left(2^{n} k_{n+1}+1\right) l_{n}>2^{n} l_{n} .
$$

Fix any $m>0$. For every $n>m$ such that $\xi_{n}=0$ and every $\alpha, \beta \in \Xi$ we obtain that

$$
d\left(\sigma^{i}\left(z^{\alpha}\right), \sigma^{i}\left(z^{\beta}\right)\right)<2^{-m}
$$

provided that $i \in Q \cap\left[l_{n}, l_{n+1}\right)=\left[l_{n}, l_{n+1}-n\right) \cap \mathbb{N}$. This shows that

$$
K \times K \subset\left[Q_{m}\right]\left(\Delta_{2^{-m}}, \sigma \times \sigma\right) \subset\left[Q_{m}\right]\left(\Delta_{1 / m}, \sigma \times \sigma\right)
$$


where $Q_{m}=Q \cap\left[l_{m},+\infty\right)$. To see that $Q \in \mathscr{M}(1)$ (which obviously implies that $\left.Q_{m} \in \mathscr{M}(1)\right)$ it is enough to calculate

$$
\begin{aligned}
\mathscr{D}^{*}(Q) & \geq \limsup _{n \rightarrow \infty} \frac{1}{l_{n+1}} \#\left(Q \cap\left[l_{n}, l_{n+1}-n\right)\right) \\
& \geq \lim _{n \rightarrow \infty} \frac{l_{n+1}-l_{n}-n}{l_{n+1}} \geq 1-\lim _{n \rightarrow \infty} \frac{2 l_{n}}{l_{n+1}} \\
& \geq 1-\lim _{n \rightarrow \infty} \frac{2 l_{n}}{2^{n} l_{n}}=1 .
\end{aligned}
$$

Next, if we fix any $\alpha, \beta \in \Xi$ then for every $m$ there is $n>m$ such that $\alpha[n] \neq \beta[n]$. But then we see that

$$
\begin{aligned}
\#\left\{0 \leq i<l_{n+1}:\right. & \left.d\left(\sigma^{i}\left(z^{\alpha}\right), \sigma^{i}\left(z^{\beta}\right)\right)>2 \varepsilon\right\} \\
\geq & \#\left\{0 \leq i<l_{n+1}: z_{i}^{\alpha} \neq z_{i}^{\beta}\right\} \\
\geq & \#\left\{l_{n} \leq i<l_{n+1}: z_{i}^{\alpha} \neq z_{i}^{\beta}\right\} \\
& \geq \#\left\{l_{n} \leq i<l_{n+1}: A_{n+1}[i] \neq \bar{A}_{n+1}[i]\right\} \\
& \geq\left|A_{n+1}\right| \eta(n+1) \geq\left|A_{n+1}\right| \theta \\
& \geq\left(l_{n+1}-l_{n}\right) \theta .
\end{aligned}
$$

This immediately implies that

$$
\begin{aligned}
\mathscr{D}^{*}\left(\left\{i:\left(\sigma^{i}(x), \sigma^{i}(y)\right) \notin \Delta_{\varepsilon}\right\}\right) & \geq \lim _{n \rightarrow \infty} \frac{l_{n+1}-l_{n}}{l_{n+1}} \theta \\
& \geq \theta\left(1-\lim _{n \rightarrow \infty} \frac{l_{n}}{2^{n} l_{n}}\right) \\
& \geq \theta>a
\end{aligned}
$$

and so $(x, y) \in \mathscr{M}(a)\left(X_{a} \times X_{a} \backslash \Delta_{\varepsilon}, \sigma \times \sigma\right)$.

REMARK 3.6. - It follows from the statement of Theorem 3.5, that $\langle Q\rangle \subset$ $\mathscr{M}(1)$ and

$$
K \times K \backslash \Delta \subset\langle Q\rangle^{+}(\Delta, \sigma \times \sigma) \cap \mathscr{M}(a)\left(X_{a} \times X_{a} \backslash \Delta_{\varepsilon}, \sigma \times \sigma\right) .
$$

Theorem 3.7. - For every $a \in[0,1)$, every $\varepsilon<1 / 2$ and every $\delta>0$ the subshift $X_{a} \subset\{0,1\}^{\mathbb{N}}$ is minimal and the shift map $\sigma=\left.\sigma\right|_{X_{a}}$ is $(\mathscr{M}(1), \mathscr{M}(a))-\varepsilon$-chaotic but is not $(\mathscr{M}(1), \mathscr{M}(1))$-chaotic. Additionally $\mathcal{H}_{d}\left(X_{a},\{0,1\}^{\mathbb{N}}\right)<\delta$.

Proof. - Fix $m$ such that $2^{-m}<\delta$ and let $v_{1}, \ldots, v_{s}$ be all possible words over $\{0,1\}$ and with length $m$. Next use $u=v_{1} v_{2} \cdots v_{s}$ in the construction of $X_{a}$.

The proof is finished by Lemma 3.3, Theorem 3.4, Theorem 3.5 and the fact that $X_{a} \cap C\left[v_{i}\right] \neq \varnothing$ for $i=1, \ldots, s$. 
We are going to show that dynamics on $X_{a}$ is to some extent similar to that of an odometer. First, we have to prove two auxiliary results. The first one seems to be well known, however we don't know any direct reference.

Lemma 3.8. - Assume that $X$ is compact and $f \in C(X)$. The following conditions are equivalent:

1. $f$ is weakly mixing,

2. there is $x$ with dense orbit such that for any open set $U \ni x$ there is $s=s_{U}>0$ such that $s, s+1 \in N(U, U, f)$.

Proof. - The proof is elementary. We leave it to the reader.

Recall that a closed set is called regular closed if it is the closure of an open set. A characteristic feature of a transitive map $f \in C(X)$ such that $f^{n}$ is not totally transitive is that $X$ may be written in an essentially unique way as the union of a finite set $\left\{D_{0}, \ldots, D_{n}\right\}$ of non-empty regular closed sets with pairwise disjoint interiors such that $f\left(D_{i}\right) \subseteq D_{i+1}(\bmod n)$ for each $i \leq n-1$. It is easy to check that these regular periodic decompositions have the property that $f^{n}$ is invariant and transitive on each $D_{i}$. Recall that a regular periodic decomposition is terminal if it is of maximal length among all periodic decomposition. If a decomposition of length $n$ is terminal then $f^{n}$ is totally transitive on each of its elements (see [6, Theorem 3.1]).

If $f$ is minimal then elements of a regular periodic decomposition are pairwise disjoint [6, Lemma 2.7]. Furthermore, we have the following property (see [6, Theorem 4.4]):

Theorem 3.9. - Let $X$ be a compact metric space and let $f \in C(X)$ be minimal. If $f$ has no terminal decomposition then $f$ is an extension of an odometer.

Following [18], we will say that $f$ has dense small periodic sets if for any nonempty open set $U$ there is a nonempty closed set $A \subset U$ and $n$ such that $f^{n}(A)=A$. It was proved in [24] that totally transitive map with dense small periodic sets is weakly mixing. It is also easy to see that if $f$ has dense small periodic sets, then so does $f^{n}$ restricted to any element of regular periodic decomposition of length $n$.

Lemma 3.10. - For every $a \in[0,1)$, the subshift $X_{a}$ constructed above has dense small periodic sets. 
Proof. - Fix any nonempty open set $U$ and denote $X=X_{a}$. There is $w$ such that $C_{X}[w] \subset U$. The orbit of $x_{a}$ is dense, so there is $m$ such that $w$ is a subword of $A_{1} \cdots A_{m} \in \mathscr{P}_{m}$. But this implies that $w$ is also a subword of $P_{1}^{(m+1)} \in$ $\mathscr{P}_{m+1}$ where $P_{1}^{(m+1)}$ is the first word in the ordering of $\mathscr{P}_{m+1}$ fixed during the construction. But $P_{1}^{(m+1)}$ is a prefix of both $A_{m+2}$ and $\overline{A_{m+2}}$. This implies that $R_{[k, k+j)}=P_{1}^{(m+1)}$ for every $R \in \mathscr{P}_{m+2}$ where $k=\left|A_{1}\right|+\cdots+\left|A_{m+1}\right|$ and $j=\left|P_{1}^{(m+1)}\right|$. The proof is finished by Lemma 3.1 and [18, Proposition 3.2].

TheOREm 3.11. - For every $a \in[0,1)$, the subshift $X_{a}$ constructed above is an extension of an odometer.

Proof. - It is enough to show that $f$ has no terminal regular periodic decomposition. Assume on contrary that such a decomposition exists, let say of length $m$, and let $D$ be a set in this decomposition so that $x_{a} \in D$. Then $D$ is invariant for $f^{m}$ and $\left.f^{m}\right|_{D}$ has dense small periodic sets by Lemma 3.10 ( $D$ is the closure of its interior by the definition). Furthermore $\left.f^{m}\right|_{D}$ is totally transitive ( $D$ is a member of terminal decomposition) which implies that $\left.f^{m}\right|_{D}$ is weakly mixing [24, Proposition 3.7]. There is $l>m$ and $R \in \mathscr{P}_{l}$ such that $W=C_{X}[R] \subset D$ and by Lemma 3.8 there is $s$ such that $s, s+m \in N(W, W, f)$. But then there are subwords $x, y$ of $x_{a}$ of length $|x|+m=|y|$ such that $R$ is a prefix and a suffix of both $x$ and $y$. Such a situation is impossible by Lemma 3.2, since $|R| \geq l>m$. We arrived to a contradiction and so the proof is finished by Theorem 3.9.

QUESTION 1. - It is not known to the author, whether $X_{a}$ are almost 1-1 extensions of odometers. By [9, Theorem 5.1] it is sufficient to prove that there is a regularly recurrent point $z \in X_{a}$, but we are not sure if $X_{a}$ must contain such a point.

We also remark here that a similar technique to [31] was used in [22] to provide an example of a minimal subshift which is distributionally chaotic. Later, even an uncountable family of such sets was obtained in [23]. We will investigate this technique later, in Section 9

In next section we will show that almost 1-1 extensions of odometers are never $(\mathscr{M}(1), \mathscr{M}(1))$-chaotic. If we could show that there is a factor map from $X_{a}$ into an odometer with a singleton fibre then it would simplify some of our arguments. However, as we mentioned above we are not sure if such a factor map exists.

TOME $140-2012-\mathrm{N}^{\mathrm{O}} 3$ 


\section{Extensions of minimal systems}

THEOREM 4.1. - Let $(X, d),(Y, \rho)$ be compact metric spaces and let $\pi: X \rightarrow$ $Y$ be a factor map between $f \in C(X)$ and $g \in C(Y)$. If $g$ is a minimal map, there is $z \in Y$ such that $\# \pi^{-1}(\{z\})=1$ and $\mathscr{F}^{+}(\Delta, g \times g)=\Delta$ for some proper family $\mathscr{F}$ then for any $\gamma>0$ we have

$$
\mathscr{F}^{+}(\Delta, f \times f) \cap \tau \mathscr{B}\left(X \times X \backslash \Delta_{\gamma}, f \times f\right)=\varnothing .
$$

In particular it implies that $f$ is not $(\mathscr{F}, \tau \mathscr{B})$-chaotic.

Proof. - We are going to show that

$$
\mathscr{F}^{+}(\Delta, f \times f) \cap \tau \mathscr{B}\left(X \times X \backslash \Delta_{\gamma}, f \times f\right)=\varnothing .
$$

for any $\gamma>0$. Fix $\gamma>0$ and consider two points $x, y \in X$.

We may assume that $f^{j}(x) \neq f^{j}(y)$ for all $j \geq 0$, since $(z, z) \notin \mathscr{F}(X \times X \backslash$ $\left.\Delta_{\gamma}, f \times f\right)$ for any $z \in X$. First assume that $\pi\left(f^{j}(x)\right) \neq \pi\left(f^{j}(y)\right)$ for every $j \geq 0$. For any $n$ there is $\delta_{n}>0$ such that $(\pi \times \pi)\left(\Delta_{\delta}\right) \subset \Delta_{1 / n}$, where $\Delta_{\delta}$ and $\Delta_{1 / n}$ are neighborhoods of diagonals in $X \times X$ and $Y \times Y$ respectively. But then

$$
N\left((x, y), \Delta_{\delta_{n}}, f \times f\right) \subset N\left((\pi(x), \pi(y)), \Delta_{1 / n}, g \times g\right)
$$

which in other words means that we have the implication

$$
(\pi(x), \pi(y)) \notin \mathscr{F}^{+}(\Delta, g \times g) \quad \Longrightarrow \quad(x, y) \notin \mathscr{F}^{+}(\Delta, f \times f) .
$$

This ends the proof for this case.

For the second case, without loss of generality, we may assume that $\pi(x)=$ $\pi(y)$, say $x, y \in \pi^{-1}(\{q\})$ for some $q \in X$. Let $p \in X$ denote the unique point $\pi(p)=z$. There is an open set $U \ni p$ such that $U \times U \subset \Delta_{\gamma} \subset X \times X$. There is also an open set $V \ni z$ such that $\pi^{-1}(V) \subset U$ as otherwise $\pi^{-1}(\{z\}) \neq\{p\}$. The point $\pi(x)$ is uniformly recurrent and $g^{m}(\pi(x)) \in V$ for some $m \geq 0$. This implies that there is a syndetic set $F$ such that $N(\pi(x), V, g)=F$, and so

$$
\begin{aligned}
N\left((x, y), \Delta_{\gamma}, f \times f\right) & \supset N((x, y), U \times U, f \times f) \\
& \supset N((\pi(x), \pi(y)), V \times V, g \times g) \\
& =N(\pi(x), V, g)=F .
\end{aligned}
$$

Then $N\left((x, y), \Delta_{\gamma}, f \times f\right) \in k \tau \mathscr{B}$, which implies that $N\left((x, y), X \times X \backslash \Delta_{\gamma}, f \times\right.$ $f) \notin \tau \mathscr{B}$, or equivalently $(x, y) \notin \tau \mathscr{B}\left(X \times X \backslash \Delta_{\gamma}, f \times f\right)$.

Corollary 4.2. - Let $(X, d),(Y, \rho)$ be compact metric spaces and let $\pi$ : $X \rightarrow Y$ be a factor map between $f \in C(X)$ and $g \in C(Y)$. If $g$ is minimal, distal and there is $x \in Y$ such that $\# \pi^{-1}(\{x\})=1$ then for any $\gamma>0$ and any proper family $\mathscr{F}$ we have

$$
\mathscr{F}^{+}(\Delta, f \times f) \cap \tau \mathscr{B}\left(X \times X \backslash \Delta_{\gamma}, f \times f\right)=\varnothing .
$$


In particular, $f$ is not $(\mathscr{F}, \tau \mathscr{B})$-chaotic for any proper family $\mathscr{F}$ and does not have DC1 pairs (thus is not $(\mathscr{M}(1), \mathscr{M}(1))$-chaotic).

Proof. - First note that $\mathscr{M}(1) \subset \tau \mathscr{B}$. By the definition of distality, for every $y, z \in Y$ there is $\delta>0$ such that $\left(g^{n}(y), g^{n}(z)\right) \notin \Delta_{\delta}$ for any $n$. Then $(y, z) \notin$ $\mathscr{F}^{+}(\Delta, g \times g)$ for any proper family $\mathscr{F}$ and so the result follows by Theorem 4.1.

As a consequence of Corollary 4.2 we see that every minimal map with a regularly recurrent point cannot have DC1 pairs (all such maps are almost 1-1 extensions of odometers [9, Theorem 5.1.]). This fact was first proved in [4, Theorem 2].

Corollary 4.3. - Let $(X, d),(Y, \rho)$ be compact metric spaces and let $\pi$ : $X \rightarrow Y$ be a factor map between $f \in C(X)$ and $g \in C(Y)$. If $g$ is a minimal map, there is $z \in Y$ such that $\# \pi^{-1}(\{z\})=1$ and $(x, y) \in \tau \mathscr{B}\left(X \times X \backslash \Delta_{\gamma}, f \times f\right)$ for some $\gamma>0$ then $x, y$ belong to different fibres of $\pi$, that is $\pi(x) \neq \pi(y)$.

Proof. - It is explicitly calculated in the second half of the proof of Theorem 4.1.

REMARK 4.4. - Almost 1-1 extensions of distal systems, for instance those of irrational rotations or odometers, fulfill the assumptions of Corollary 4.2.

\section{Toeplitz flows}

In this section we will construct another family of systems with positive topological entropy which are not $(\mathscr{M}(1), \mathscr{M}(1))$-chaotic. The construction will be less transparent than this in Section 3, however it will allow us to raise the topological entropy above zero (in fact, arbitrarily close to the entropy of the full shift). There is well known technique of construction of such systems and it allows to 'pack' any subshift in the aperiodic readouts of the resulting Toeplitz system (e.g. see WilliamsŠ Construction on page 30 of [9]). Nevertheless, we decided to perform a construction of a special family of Toeplitz flows to highlight the fact that the edge between $(\mathscr{M}(1), \mathscr{M}(1))$-chaos and $(\mathscr{M}(1), \mathscr{M}(a))$-chaos can be much more thick than can be foreseen by the application of Corollary 4.2. Strictly speaking, for any $0<a<b<1$ we will obtain a system which is $(\mathscr{M}(1), \mathscr{M}(a))$-chaotic but is not $(\mathscr{M}(1), \mathscr{M}(b))$ chaotic, or even more

$$
\mathscr{M}(1)^{+}(\Delta, \sigma \times \sigma) \cap \mathscr{M}(b)\left(X_{\omega} \times X_{\omega} \backslash \Delta_{\gamma}, \sigma \times \sigma\right)=\varnothing
$$

for any $\gamma>0$. Unfortunately, systems obtained by this method are never mixing or even weakly mixing, since they have a nontrivial equicontinuous factor (see the proof of [20, Prop. 2.45]). 
First, let us recall some more facts on Toeplitz flows. Suppose that a Toeplitz flow $X_{\omega}$ is an extension of an odometer $G_{\mathbf{s}}$ via a factor map $\pi_{\omega}$. If we fix any $\mathbf{j} \in G_{\mathbf{s}}$ then $\operatorname{Aper}(x)=\operatorname{Aper}(y)$ for every $x, y \in \pi_{\omega}^{-1}(\mathbf{j})$; in particular we can write $\operatorname{Aper}(\mathbf{j}):=\operatorname{Aper}(x)$. Assume that $\operatorname{Aper}(x)$ is infinite and enumerate its elements $\operatorname{Aper}(x)=\left\{0 \leq n_{1}<n_{2}<\cdots\right\}$. Then by the aperiodic readout of $x$ we mean the sequence

$$
y:=\left.x\right|_{\operatorname{Aper}(x)}=x\left[n_{1}\right] x\left[n_{2}\right] \cdots
$$

In other words, we read and write down symbols along $\operatorname{Aper}(x)$. For every $\mathbf{j} \in G_{\mathbf{s}}$ with \# $\operatorname{Aper}(\mathbf{j})=\infty$, let $Y_{\mathbf{j}}$ be the set of all possible aperiodic readouts of elements in $\pi_{\omega}^{-1}(\mathbf{j})$.

ThEOREM 5.1. - Assume that $X_{\omega}$ is a Toeplitz flow and there is $\mathbf{j}$ such that $\mathscr{D}(\operatorname{Aper}(\mathbf{j}))=\eta>0$. If $Y_{\mathbf{j}}$ contains an $(\mathscr{M}(1), \mathscr{M}(a))-\gamma$-scrambled set for some $a>0, \gamma \in(0,1 / 2)$ then $X_{\omega}$ contains $\left(\mathscr{M}(1), \mathscr{M}\left(a^{\prime}\right)\right)-1 / 2$-scrambled set, where $a^{\prime}=a \gamma \eta$.

Proof. - Fix $m>0$ such that $2^{-m}<\gamma \leq 2^{-m+1}$ and observe that if $\left(\sigma^{i}(p), \sigma^{i}(q)\right) \notin \Delta_{\gamma}$ then $d\left(\sigma^{i}(p), \sigma^{i}(q)\right) \geq \gamma$ and so $p_{[i, i+m)} \neq q_{[i, i+m)}$. Take $p, q \in Y_{\mathbf{j}}$ such that

$$
(p, q) \in \mathscr{M}(1)^{+}(\Delta, f \times f) \cap \mathscr{M}(a)\left(X_{\omega} \times X_{\omega} \backslash \Delta_{\gamma}, \sigma \times \sigma\right)
$$

and let $x, y$ be points in the fibre defined by $\mathbf{j}$ with aperiodic readouts $p$ and $q$ respectively.

For any $\delta>0$ we can find arbitrarily large $n$ such that we have

$$
\begin{aligned}
a-\delta & \leq \frac{1}{n} \#\left\{0 \leq i<n: p_{[i, i+m)} \neq q_{[i, i+m)}\right\} \\
& \leq \frac{m}{n} \#\{0 \leq i<n: p[i] \neq q[i]\} .
\end{aligned}
$$

Then we obtain that

$$
\gamma(a-\delta) \leq \frac{a-\delta}{2^{m-1}} \leq \frac{a-\delta}{m} \leq \frac{1}{n} \#\{0 \leq i<n: p[i] \neq q[i]\} .
$$

Additionally, observe that $x[i] \neq y[i]$ if and only if $i=n_{j}$ for some $n_{j} \in \operatorname{Aper}(\mathbf{j})$ $\left(n_{j}\right.$ is $j$-th element in this set) and $p[j] \neq q[j]$. Given $n>0$ let $n^{\prime}$ denote $\#(\operatorname{Aper}(\mathbf{j}) \cap[0, n))$. By the above observations we obtain that for any $\delta>0$ and sufficiently large $n=n(\delta)$ we have the following lower bound

$$
\begin{aligned}
\frac{1}{n} \#\{0 \leq i<n: x[i] \neq y[i]\} & =\frac{1}{n} \#\left\{0 \leq j<n^{\prime}: p[j] \neq q[j]\right\} \\
& \geq \frac{1}{n}(\eta-\delta) n \gamma(a-\delta) .
\end{aligned}
$$

Since $\delta$ can be arbitrary small, we see that

$$
(p, q) \in \mathscr{M}(a \eta \gamma)\left(X_{\omega} \times X_{\omega} \backslash \Delta_{1 / 2}, \sigma \times \sigma\right) .
$$


Next, observe that for any $k>0$, if

$$
\frac{1}{n} \#\left\{i<n: p_{[i, i+k)} \neq q_{[i, i+k)}\right\}<\varepsilon
$$

then

$$
\frac{1}{n} \#\{i<n: p[i] \neq q[i]\}<\varepsilon .
$$

Furthermore, if $n$ is such that aperiodic readout of $x$ over $x_{[0, n)}$ contains exactly $n^{\prime}$ symbols then for sufficiently large $n$ we have $n^{\prime} / n<2 \eta$ and so

$$
\frac{1}{n} \#\{0 \leq i<n: x[i] \neq y[i]\}<\varepsilon \frac{n^{\prime}}{n} \leq 2 \varepsilon \eta
$$

But if $x[i] \neq y[i]$ then also $x_{[j, j+l)} \neq y_{[j, j+l)}$ for any $l>0$ and any $j$ such that $i \in[j, j+l)$, which gives

$$
\frac{1}{n} \#\left\{0 \leq i<n: x_{[i, i+l)}=y_{[i, i+l)}\right\}>1-2 l \varepsilon \eta-\frac{l}{n} .
$$

provided that (5.1) is satisfied. By the above observation and the fact that $\varepsilon$ can be arbitrarily small (and does not depend on $l$ ) we see that also for any fixed $l$ we have

$$
\limsup _{n \rightarrow \infty} \frac{1}{n} \#\left\{0 \leq i<n: x_{[i, i+l)}=y_{[i, i+l)}\right\}=1
$$

and so the proof is finished.

REMARK 5.2. - There is a well known technique (which originated from papers of Susan Williams [32]) of construction of Toeplitz flows fulfilling assumptions of Theorem 5.1 (see [9, p. 30]).

The following definition is a stronger version of the same property introduced in [9].

Definition 5.3. - We say that $X_{\omega}$ fulfils the condition $(S A R)$ if $Y_{\mathbf{j}}$ are the same for every $\mathbf{j} \in G_{\mathbf{s}}$ with $\# \operatorname{Aper}(\mathbf{j})=\infty$.

Theorem 5.4. - For every $a \in[0,1)$, every $b \in(a, 1]$, every $\varepsilon<1$ there is a Toeplitz subshift $X_{a} \subset\{0,1\}^{\mathbb{N}}$ fulfilling (SAR) such that the shift map $\sigma=$ $\left.\sigma\right|_{X_{a}}$ is $(\mathscr{M}(1), \mathscr{M}(a))-\varepsilon$-chaotic but is not $(\mathscr{M}(1), \mathscr{M}(b))$-chaotic. Additionally $h_{\text {top }}\left(X_{a}\right)=a$.

Proof. - Start with the construction of two sequences $s_{m}, s_{m}^{\prime}$. Put $s_{0}=s_{0}^{\prime}=1$ and next $s_{m+1}=s_{m} q_{m}, s_{m+1}^{\prime}=s_{m}^{\prime} q_{m}^{\prime}$, where $q_{0}^{\prime} / q_{0} \in(a, b), q_{m}>q_{m}^{\prime}>2$ and $\lim _{m \rightarrow \infty} s_{m}^{\prime} / s_{m}=a$. Such sequence can be easily constructed by induction, e.g. in the following way. Denote $\alpha(m)=\log \left(s_{m}^{\prime}\right)-\log \left(s_{m}\right)-\log (a)$ and observe that for the construction it is enough, if $\lim _{m \rightarrow \infty} \alpha(m)=0$. 
Since $\lim _{n \rightarrow \infty} \log \left(\frac{n+1}{n}\right)=0$ there is $q_{m}^{\prime}$ such that $\log \left(q_{m}^{\prime}+k+1\right)-\log \left(q_{m}^{\prime}+\right.$ $k)<\alpha(m) / 2$ for every $k \geq 0$. In particular, there is $k$ such that $\alpha(m) \geq$ $\log \left(q_{m}^{\prime}+k\right)-\log \left(q_{m}^{\prime}\right)>\alpha(m) / 2$. If we put $q_{m}=q_{m}^{\prime}+k$ then $\alpha(m+1)<\alpha(m) / 2$.

From now on, we assume that sequences $s_{m}, s_{m}^{\prime}, q_{m}, q_{m}^{\prime}$ are fixed. We put $r_{m}=q_{m}-q_{m}^{\prime}$ and $t_{m}=s_{m}^{\prime} r_{m}$. Then we fix a sequence of words $B_{m} \in\{0,1\}^{+}$ such that $\left|B_{m}\right|=t_{m}$ and every word $u \in\{0,1\}^{+}$is a prefix and a suffix of some (possibly different) words $B_{m}$. In addition to symbols 0,1 we will also use the symbol $\diamond$. If $|w|_{\diamond}=m$ and $|u|=m$ then by $w \downarrow u$ we denote the word obtained from $w$ by putting symbols of $u$ in place of $\diamond$, e.g $11 \diamond 1 \downarrow 0=1101$.

For every $n \geq 1$ we will define a word $V(n)$ such that $|V(n)|=s_{n}$ and $|V(n)|_{\diamond}=s_{n}^{\prime}$. First, we put $V(1)=B_{0} \triangleright^{s_{1}^{\prime}}$. Next, if $V(n)$ is defined, then we put $W(n)=V(n)^{r_{n}} \downarrow B_{n}$ (this operation is well defined since $V(n)^{r_{n}}$ contains $t_{n}$ occurrences of $\left.\diamond\right)$ and finally $V(n+1)=W(n) V(n)^{q_{n}^{\prime}}$.

Note that for every $n \geq 1$ and $k>1$ the word $V(n) V(n)$ is a suffix of $V(n+k)$. Let $S_{n}=V(n)^{\infty}$ and $\omega=\lim _{n \rightarrow \infty} S_{n}$, where the limit is taken in the space $\{0,1, \diamond\}^{\mathbb{N}}$. Observe that $\omega$ is a Toeplitz sequence, such that $\operatorname{Per}_{s_{n}}(\omega)=$ $\left\{i: S_{n}[i] \neq \diamond\right\}$ (the inclusion $\supset$ is obvious, and the converse follows from the definition of $\left.B_{m}\right)$. Note that

$$
\lim _{m \rightarrow \infty} \mathscr{D}\left(\operatorname{Per}_{s_{m}}(\omega)\right)=1-\lim _{m \rightarrow \infty} \frac{s_{m}^{\prime}}{s_{m}}=1-a .
$$

Additionally it follows from the construction, that for any $u \in\{0,1\}^{+},|u|=2 s_{n}$ the word $V(n) V(n) \downarrow u$ is a suffix of $W(m)$ for some $m>0$ ( $u$ is a suffix of some $\left.B_{m}\right)$. Furthermore, for every subword $u$ of $\omega$ with $|u|=s_{n}$ there is a subword $v$ of $V(n) V(n)$ such that $u$ is obtained form $v$ by replacement of all $\diamond$ by symbols 0,1 .

Note that $|v|_{\diamond}=|V(n)|_{\diamond}=s_{n}^{\prime}$. Then, similarly to the proof of [20, Theorem 4.77], we obtain that

$$
2^{s_{n}^{\prime}} \leq \# \mathscr{L}_{s_{n}}\left(X_{\omega}\right) \leq s_{n} 2^{s_{n}^{\prime}}
$$

and so

$$
h_{\mathrm{top}}\left(X_{\omega}\right)=\lim _{n \rightarrow \infty} \frac{s_{n}^{\prime}}{s_{n}} \log (2)=a .
$$

Additionally, observe that $X_{\omega}$ fulfills (SAR). Take any $\mathbf{j} \in G_{\mathbf{s}}$ with \# Aper $(\mathbf{j})=$ $\infty$ and fix any $x \in \pi_{\omega}^{-1}(\mathbf{j})$. Assume that $\operatorname{Aper}(x)=\left\{n_{1}<n_{2}<\cdots\right\}$. Fix any $k$, let $s_{m}>n_{k}$ and let $u$ be the prefix of $x$ with $|u|=s_{m}$. Then, there is $v \subset V(m) V(m)$ such that $v\left(n_{i}\right)=\diamond$ for $i=1,2, \ldots, k$. We can replace some of the symbols $u\left(n_{i}\right)$ to opposite ones, obtaining a word $u^{\prime}$. Note that $u^{\prime} \in \mathscr{L}\left(X_{\omega}\right)$ and furthermore, $u^{\prime}$ is obtained from the same symbols of $V(m) V(m)$ that previously defined $u$. But then, if we define $y \in\{0,1\}^{\mathbb{N}}$ putting $y[i]=x[i]$ for $i \notin \operatorname{Aper}(x)$, and any choice of symbols on other positions, then by the 
above comment still $y \in X_{\omega}$. Additionally, note that if $x=\lim _{n \rightarrow \infty} \sigma^{j_{m}^{\prime}}(\omega)$, where $j_{m}^{\prime}=j_{m}\left(\bmod s_{m}\right)$ and $j_{m}$ is the $m$-th coordinate of $\mathbf{j}$, then by the construction of the sequence $V(n)$ and the above remark, there is a sequence $j_{m}^{\prime \prime}=j_{m}\left(\bmod s_{m}\right)$ such that $y=\lim _{n \rightarrow \infty} \sigma^{j_{m}^{\prime \prime}}(\omega)$. This shows that $\pi_{\omega}(x)=$ $\pi_{\omega}(y)$. In particular $Y_{\mathbf{j}}=\{0,1\}^{\mathbb{N}}$ which ends the proof of (SAR).

This implies, by [9, Cor. 14.2] that there is $\mathbf{q} \in G_{\mathbf{s}}$ with $\mathscr{D}(\operatorname{Aper}(\mathbf{q}))=a$. With all the above facts, we can continue with the proof. First, if $x, y$ are in different fibres of $\pi_{\omega}$ then their orbits are not proximal, thus $(x, y) \notin$ $\mathscr{M}(1)\left(\Delta_{\gamma}, \sigma \times \sigma\right)$ for some sufficiently small $\gamma>0$. Assume that $x, y$ are in the same fiber and fix any $m>0$. For every $\delta>0,3 \delta<b-a$, there is $n$ such that $m / s_{n}<\delta$ and $s_{n}^{\prime} / s_{n}-\delta<a$. By the construction, word $W(n)$ appears in $x$ and $y$ with period $s_{n}$, so for any $l>2$ we calculate

$$
\begin{aligned}
\frac{1}{l} \#\left\{0 \leq i \leq l s_{n}: x_{[i, i+m)}=y_{[i, i+m)}\right\} & \geq \frac{(l-1)(|W(n)|-m)}{l s_{n}} \\
& \geq \frac{(l-1)}{l}\left(1-\frac{s_{n}^{\prime}}{s_{n}}-\frac{m}{s_{n}}\right) \\
& \geq \frac{(l-1)}{l}(1-a-2 \delta) .
\end{aligned}
$$

Then

$$
\mathscr{D}_{*}\left(\left\{i: d\left(\sigma^{i}(x), \sigma^{i}(y)\right) \leq 2^{-m}\right\}\right) \geq 1-a-2 \delta>1-b,
$$

in particular

$$
(x, y) \notin M(b)\left(X_{\omega} \times X_{\omega} \backslash \Delta_{\gamma}, \sigma \times \sigma\right)
$$

for any $\gamma>0$. Next, in a similar way as it was done in (3.3), we can construct a Cantor set $C \subset\{0,1\}^{\mathbb{N}}$ such that for every $n$ and every $x, y \in C$ there are $k, l$ such that $x[i]=y[i]$ for $i \in\left[k,\left(2^{n}+1\right) k\right]$ and $x[i] \neq y[i]$ for $i \in\left[l,\left(2^{n}+1\right) l\right]$. For every $x \in C$ denote by $z_{x} \in \pi^{-1}(\mathbf{q})$ the point with aperiodic readout equal to $x$. Now, if we fix any $\varepsilon<1$ and denote $K=\left\{z_{x}: x \in C\right\}$ then $K$ is a Cantor set, and we see that

$$
K \times K \backslash \Delta \subset \mathscr{M}(1)^{+}(\Delta, \sigma \times \sigma) \cap \mathscr{M}(a)\left(X_{\omega} \times X_{\omega} \backslash \Delta_{\varepsilon}, \sigma \times \sigma\right) .
$$

It is enough to put $X_{a}=X_{\omega}$ and the proof is finished.

Note that if $b<1$ then $\mathscr{M}(b) \backslash \tau \mathscr{B} \neq \varnothing$ and obviously also $\tau \mathscr{B} \backslash \mathscr{M}(b) \neq \varnothing$, since there are thick subsets with small density (even zero), and sets with high density which are not thick (e.g. complements of infinite progressions).

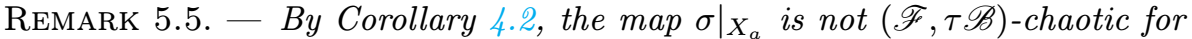
any proper family $\mathscr{F}$, since it is an almost 1-1 extension of an odometer.

TOME $140-2012-\mathrm{N}^{\mathrm{O}} 3$ 


\section{Entropy, DC1 and regularly recurrent points}

In [4] the authors state the problem whether minimal system with positive topological entropy has a DC1 pair if and only if it contains no regularly recurrent point (Problem (iii) in [4]). In this section we will prove that there is a minimal system with positive topological entropy but without DC1 pairs nor regularly recurrent points.

Consider first $F=R_{\rho} \times R_{\mathrm{s}}$ where $R_{\rho}: \mathbb{S} \rightarrow \mathbb{S}$ is an irrational rotation of the unit circle $R_{\rho}(x)=x+\rho(\bmod 1)$ and $R_{\mathrm{s}}$ is a nontrivial odometer on a scale $\mathbf{s}$. It is easy to verify that $F$ is distal, hence every point is uniformly recurrent (see [11, p. 160]). Additionally, $R_{\rho}^{n}=R_{n \rho}$ is minimal for every $n$, and every point is regularly recurrent under $R_{\mathrm{s}}$, so $F$ is also minimal. By the same argument, we see that $F$ has no regularly recurrent points.

Now, let $T$ be a Toeplitz flow with positive topological entropy, constructed as an almost 1-1 extension of the odometer $R_{\mathrm{s}}$. Then the map $G=R_{\rho} \times T$ is an almost 1-1 extension of $F$ and obviously its topological entropy is positive. But there is no regularly recurrent point under $G$, since if there were such a point for $G$ then $F$ also would have a regularly recurrent point. There is also no DC1 pair for $G$ by Corollary 4.2. Therefore we answered Problem (iii) from [4, p. 1678] in negative:

TheOREM 6.1. - There is a minimal system with positive topological entropy but without DC1 pairs and without regularly recurrent points.

\section{Minimal systems with positive entropy and u.p.e.}

Another technique originated from papers C. Grillenberger [12, 13] (see also [14]), where the first explicit (symbolic) examples of strictly ergodic systems with positive entropy were constructed. For the purposes of this section results of [13] are particularly important, since examples of minimal $K$-systems are constructed there. We will comment on this in the further parts of this section. The main result of this section is that all minimal u.p.e. systems are DC2 (and it is known that (invertible) $K$-systems are topological $K$-systems [16], in particular they are u.p.e.).

The following fact seems to be folklore (e.g. see [19, p.877]) and can be proved using the Stirling formula (we leave the calculations to the reader).

Lemma 7.1. - For every $t>0$ there are $c>0$ and $N>0$ such that

$$
\sum_{k=0}^{\lfloor c n\rfloor}\left(\begin{array}{l}
n \\
k
\end{array}\right)<2^{t n}
$$

for every $n>N$. 
LEMMA 7.2. - If $f \in C(X)$ is minimal and has positive topological entropy, then there are $\varepsilon, \alpha>0$ such that for every nonempty open set $U$ and $K>0$ there are $n>K$ and a pair of points $p, q \in U$ such that

$$
\frac{1}{n} \#\left\{0 \leq i \leq n: d\left(f^{i}(p), f^{i}(q)\right)>\varepsilon\right\}>\alpha .
$$

Proof. - First observe that by the definition of topological entropy, there are $\gamma, t>0$ such that

$$
\limsup _{n \rightarrow \infty} \frac{\log \left(S_{X}(n, \gamma)\right)}{n}=2 t>0 .
$$

Let $c$ and $N$ be provided for $t$ by Lemma 7.1. The set $X$ is compact, so it can be covered by a finite number of balls with radius $\gamma / 4$, let say $V_{1}, \ldots, V_{m}$. Decreasing $c$ if necessary, we may additionally assume that $c \log (m)<t / 2$. Fix $\alpha<c$ and let $\varepsilon>0$ be such that $2 \varepsilon$ is a Lebesgue number of the cover $\left\{V_{i}\right\}$.

Fix any open set $U$. The system $(f, X)$ is minimal, thus there is $k$ such that $\bigcup_{i=0}^{k} f^{-i}(U)=X$ and so also

$$
\limsup _{n \rightarrow \infty} \frac{\log \left(S_{U}(n, \gamma)\right)}{n}=2 t>0 .
$$

Assume that $A \subset U$ is an $(n, \gamma)$-separating set, where $n>N$. If $x, y \in A$ and $d\left(f^{i}(x), f^{i}(y)\right) \geq \gamma / 2$ then $f^{i}(x), f^{i}(y)$ cannot both belong to the same set $V_{j}$. Then the number of elements $x, y \in A$ which are not in the same set $V_{j}$ during iterations with numbers $0 \leq i_{1}<i_{2}<\cdots<i_{s}<n$ is bounded from the above by $m^{s}$ (just by the definitions of $(n, \gamma)$-separating set).

We claim that for every $N>0$ there are $n>N$ and an $(n, \gamma)$-separating set $A \subset U$ and $p, q \in A$ such that

$$
\#\{0 \leq i \leq n: \tau(q, i) \neq \tau(p, i)\}>\alpha n
$$

where $\tau(x, i)$ is the index such that $f^{i}(x) \in V_{\tau(x, i)}$. While there are probably more than one possible ways of defining functions $\tau(q, \cdot), \tau(p, \cdot)$, we ensure that $\tau(q, i)=\tau(p, i)$ for as many $i$ as possible.

In contrary, assume that there in $N>0$ such that for any $n>N$ any $(n, \gamma)$-separating set and any $p, q \in A$ we have that

$$
\#\{0 \leq i \leq n: \tau(q, i) \neq \tau(p, i)\}<\alpha .
$$

In particular, by the choice of $\alpha$ we also have

$$
\#\{0 \leq i \leq n: \tau(q, i) \neq \tau(p, i)\}<\lfloor c n\rfloor
$$

This implies that $f^{i}(p), f^{i}(q)$ can be in different sets $V_{j}$ during at most $\lfloor c n\rfloor$ iterations. If we $z \in A$ then we can uniquely identify other points of $A$ by saying that during iteration $i$ they visited the same set $V_{j}$ as $z$ or providing the label 
(i.e. index $j$ ) of the visited set when it was different. Furthermore, there is at most one point of $A$ generating given itinerary. That way we can estimate that

$$
\begin{aligned}
\# A \leq \sum_{k=0}^{\lfloor c n\rfloor} m^{k}\left(\begin{array}{l}
n \\
k
\end{array}\right) & \leq m^{c n} 2^{t n} \\
& \leq 2^{\log (m) c n} 2^{t n} \\
& <2^{3 t n / 2} .
\end{aligned}
$$

As an immediate consequence of the above we get that

$$
2 t=\limsup _{n \rightarrow \infty} \frac{\log \left(S_{U}(n, \gamma)\right)}{n}<\lim _{n \rightarrow \infty} \frac{\log \left(2^{3 t n / 2}\right)}{n}<2 t
$$

which is impossible. Indeed, the claim must be satisfied.

But then for $n>N$ and $p, q$ specified in the above claim we have

$$
\begin{aligned}
\frac{1}{n} \#\left\{0 \leq i \leq n: d\left(f^{i}(p), f^{i}(q)\right)>\varepsilon\right\} & \geq \frac{1}{n} \#\{0 \leq i \leq n: \tau(q, i) \neq \tau(p, i)\} \\
& >\alpha .
\end{aligned}
$$

and so the proof is finished.

THEOREM 7.3. - If $f \in C(X)$ is a minimal system with positive topological entropy then there are $a, \gamma>0$ such that the set

$$
\mathscr{M}(a)\left(X \times X \backslash \Delta_{\gamma}, f \times f\right)
$$

is residual in $X \times X$.

Proof. - Let $\varepsilon, \alpha>0$ be provided by Lemma 7.2. Denote $a=\alpha / 6$ and $\gamma=\varepsilon / 4$. For any $m>0$ define the set $Q_{m} \subset X \times X$ in the following way. A pair $(x, y) \in Q_{m}$ if there is $n>m$ such that

$$
\frac{1}{n} \#\left\{0 \leq i<n: d\left(f^{i}(x), f^{i}(y)\right)>2 \gamma\right\}>2 a-\frac{1}{m} .
$$

The map $f$ is uniformly continuous, and so it is immediate that $Q_{m}$ is open. By Lemma 7.2 it is also dense, since for any $z \in X$ and $p, q \in X$

$$
\frac{1}{n} \#\left\{0 \leq i<n: d\left(f^{i}(p), f^{i}(z)\right)>2 \gamma\right\}>2 a
$$

or

$$
\frac{1}{n} \#\left\{0 \leq i<n: d\left(f^{i}(q), f^{i}(z)\right)>2 \gamma\right\}>2 a
$$

provided that

$$
\frac{1}{n} \#\left\{0 \leq i<n: d\left(f^{i}(q), f^{i}(p)\right)>\varepsilon\right\}>\alpha-a .
$$


This shows that the set $Q_{a}=\bigcap_{m=k}^{\infty} Q_{m}$ is residual, where $k$ is such that $k>1 / a$. Now, if $(x, y) \in Q_{a}$ then there are $n_{k}<n_{k+1}<\cdots$ such that for every $j \geq k$ we have

$$
\frac{1}{n_{j}} \#\left\{0 \leq i<n_{j}: d\left(f^{i}(x), f^{i}(y)\right)>2 \gamma\right\}>2 a-\frac{1}{j}>a .
$$

This immediately implies that $\mathscr{D}^{*}(F) \geq a$, where

$$
F=\left\{i:(f \times f)^{i}(x, y) \in X \times X \backslash \Delta_{\gamma}\right\}
$$

and so $F \in \mathscr{M}(a)$. The proof is completed.

THEOREM 7.4. - If $f \in C(X)$ is u.p.e. then

$$
\mathscr{M}(1)^{+}(\Delta, f \times f)
$$

is residual in $X \times X$.

Proof. - Fix $m>0$ and define a set $A_{m}$ in the following way: $(x, y) \in A_{m}$ if there is $n>m$ such that

$$
\frac{1}{n} \#\left\{0 \leq i<n:\left(f^{i}(x), f^{i}(y)\right) \in \Delta_{1 / m}\right\}>1-\frac{1}{m} .
$$

Again, it is easy to verify that each set $A_{m}$ is open. If $f$ is invertible then $A_{m}$ is also dense, because the set of asymptotic pairs is dense in $X \times X$ in every invertible u.p.e. system by [7, Proposition 4]. In the case that $f$ is not homeomorphism the argument is the following. Let us consider natural extension of $f$ to the shift map $\sigma_{f}$ on the inverse limit of $X$ with $f$ as the single bounding map, that is $\sigma_{f}: \mathbb{X}_{f} \rightarrow \mathbb{X}_{f}$, where $\mathbb{X}_{f}=\left\{\left(x_{1}, x_{2}, \cdots\right) \in \prod_{1}^{\infty} X: f\left(x_{n+1}\right)=x_{n}, n \in \mathbb{N}\right\}$ and $\sigma_{f}\left(x_{1}, x_{2}, \cdots\right)=\left(f\left(x_{1}\right), x_{1}, \cdots\right)$. Next, by [15, Theorem 4.1] we get that $\sigma_{f}$ is also u.p.e. and so we are allowed to use [7, Proposition 4], thus asymptotic pairs of $\sigma_{f}$ are dense in $\mathbb{X}_{f} \times \mathbb{X}_{f}$. But asymptotic pairs of $\sigma_{f}$ project onto asymptotic pairs of $f$, and so $A_{m}$ is dense also in non-invertible case.

Furthermore, we can easily verify that if $(x, y) \in \bigcap_{m=1}^{\infty} A_{m}$ then

$$
\mathscr{D}^{*}\left(\left\{i:\left(f^{i}(x), f^{i}(y)\right) \in \Delta_{\varepsilon}\right\}\right)=1
$$

for every $\varepsilon>0$. In other words $(x, y) \in \mathscr{M}(1)\left(\Delta_{\varepsilon}, f \times f\right)$ and so $\mathscr{M}(1)\left(\Delta_{\varepsilon}, f \times f\right)$ is residual for every $\varepsilon>0$ which ends the proof.

Recall that a set is a Mycielski set if it can be presented as at countable union of Cantor sets. The following fact is a simplified version of Mycielski theorem (see [2]).

THEOREM 7.5. - If $X$ is perfect and $R \subset X \times X$ is symmetric and residual then there is a dense Mycielski set $M$ such that $M \times M \subset R \cup \Delta$.

TOME $140-2012-\mathrm{N}^{\mathrm{O}} 3$ 
Corollary 7.6. - If $f$ is a minimal u.p.e. then there is dense Mycielski $(\mathscr{M}(1), \mathscr{M}(a))$ - $\varepsilon$-scrambled set for some $a, \varepsilon>0$.

Proof. - Combining Theorems 7.3 and 7.4 we obtain $a, \varepsilon>0$ such that the following set is residual:

$$
\mathscr{M}(1)^{+}(\Delta, f \times f) \cap \mathscr{M}(a)\left(X \times X \backslash \Delta_{\gamma}, f \times f\right) .
$$

Now it is enough to apply Theorem 7.5 and the proof is finished.

If $f$ is (invertible) $K$-system then it is topological $K$-system by [16]. It can also be proved (see [17]) that every minimal topological K-system is mixing.

REMARK 7.7. - By Corollary 7.6 we see that there are numerous examples of minimal mixing DC2 systems with positive topological entropy. This class contains every minimal K-system, e.g. these constructed by Grillenberger in [13].

\section{Some extensions of the Chacón flow}

GrillenbergerŠs ideas were further developed to a technique, which is often called "construction by (embedded) $k$-blocks" (e.g. see [8]). This technique permits to construct weakly mixing strictly ergodic systems with positive topological entropy having a factor with zero topological entropy. In this construction, we lose some freedom when compared to extensions of Toeplitz flows, which is tribute we have to pay for embedding much complex dynamics (as we mentioned it earlier, Toeplitz flows are never weakly mixing). In this section we will investigate the class of extensions of the Chacón flow introduced in [8] from the point of view of $\mathrm{DC} 2$ pairs.

We start recalling briefly the construction of Blanchard and Kwiatkowski. For more detailed description we refer to [8]. First we present how the Chacón flow is constructed. Define a family of words $\hat{B}_{n}$ over the alphabet $\{\diamond, *\}$ by the following inductive formula. Put $\hat{B}_{0}=\diamond$ and $\hat{B}_{n+1}=\hat{B}_{n} \hat{B}_{n} * \hat{B}_{n}$ for $n \geq 0$. Note that $\left|\hat{B}_{n}\right|_{\diamond}=3^{n}$ and $d_{n}=\left|\hat{B}_{n}\right|=\frac{1}{2}\left(3^{n+1}-1\right)$. We define bi-infinite sequence $x \in\{\diamond, *\}^{\mathbb{Z}}$ by

$$
x_{\left[-d_{n}, d_{n}\right)}=\hat{B_{n}} \hat{B_{n}} .
$$

Next, we define $X=\overline{\operatorname{Orb}(x, \sigma)}$ and call the system $\left(X,\left.\sigma\right|_{X}\right)$ the Chacón flow (or Chacón subshift).

Now we are ready to construct a class of extensions of the Chacón flow introduced in [8]. Assume that $1 \leq k_{0}<k_{1}<\cdots$ are positive integers and denote

$$
p_{n}=k_{0}+\cdots+k_{n}, \quad B_{n}=\hat{B}_{p_{n}}, \quad l_{n}=\left|B_{n}\right| .
$$


We will construct a subshift over the alphabet $\mathscr{G}=\left\{1,2, \ldots, 3^{k_{0}}, *\right\}$. First, we are going to define a family $\mathscr{G}_{n}$ of blocks of length $l_{n}$ and we will denote $m_{n}=\# A_{n}$. By $\Pi(n)$ we will denote the family of permutations of the set $\{1, \ldots, n\}$.

First, fix $\sigma \in \Pi\left(3^{k_{0}}\right)$ and define $B_{\sigma}^{(1)}$ by substituting successive occurrences of $\diamond$ in $B_{0}=\hat{B}_{k_{0}}$ by $\sigma(1), \sigma(2), \ldots, \sigma\left(3^{k_{0}}\right)$. We keep the letter '*' unchanged. In the notation os Section 5 we simply define

$$
B_{\sigma}^{(1)}=B_{0} \downarrow \sigma(1) \cdots \sigma\left(k_{0}\right) .
$$

That way we can define the following set

$$
\mathscr{G}_{1}=\left\{B_{\sigma}^{(1)}: \sigma \in \Pi\left(3^{k_{0}}\right)\right\} .
$$

Next, assume that sets $\mathscr{G}_{1}, \ldots, \mathscr{G}_{n}$ are defined, and numbers $k_{0}<k_{1}<\cdots<k_{n}$ satisfy $3^{k_{i}}-2>m_{i-1}$ for $i=1, \ldots, n$, where $m_{0}=0$. Enumerate elements of $\mathscr{C}_{n}$, let say

$$
\mathscr{G}_{n}=\left\{B_{1}^{(n)}, \ldots, B_{m_{n}}^{(n)}\right\} .
$$

Fix any two different blocks $L_{n}, F_{n} \in \mathscr{G}_{n}$ and choose $k_{n+1}>m_{n}+2$. Let $t_{n+1}$ be such that

$$
t_{n+1} m_{n} \leq 3^{k_{n+1}}-2<\left(t_{n+1}+1\right) m_{n}
$$

Let $\Phi_{n}$ denote the set of functions $\phi:\left\{1, \ldots, t_{n+1} m_{n}\right\} \rightarrow\left\{1, \ldots, m_{n}\right\}$ such that each fiber has the same number of elements, that is $\# \phi^{-1}(t)=t_{n+1}$ for every $t \in\left\{1, \ldots, m_{n}\right\}$. By the inductive formula in the construction of the Chacón flow, it is possible to present $B_{n+1}$ as a concatenation of blocks $B_{n}$ with additional occurrences of the symbol ' $*$ ' somewhere between them. Note that we have exactly $3^{k_{n+1}}$ occurrences of $B_{n}$ in $B_{n+1}$. Fix any $\sigma \in \Phi_{n}$ and denote $u_{n}=3^{k_{n+1}}-t_{n+1} m_{n}-2 \geq 0$. We define the block $B_{\sigma}^{(n+1)}$ by substituting first $u_{n}+1$ occurrences of $B_{n}$ in $B_{n+1}$ by the block $L_{n}$. Next $t_{n+1} m_{n}$ occurrences of $B_{n}$ are replaced by $B_{\sigma(j)}^{(n)}, j=1, \ldots, t_{n+1} m_{n}$, and finally we replace the last remaining occurrence of $B_{n}$ by the block $F_{n}$. In other words (here we substitute whole $B_{n}$, not single occurrences of $\left.\diamond\right)$ :

$$
B_{\sigma}^{(n+1)}=B_{n+1} \downarrow\left(L_{n}\right)^{u_{n}+1} B_{\sigma(1)}^{(n)} \cdots B_{\sigma\left(t_{n+1 m_{n}}\right)}^{(n)} F_{n} .
$$

That way we obtain the following set of blocks

$$
\mathscr{G}_{n+1}=\left\{B_{\sigma}^{(n+1)}: \sigma \in \Phi_{n}\right\} .
$$

We continue with this construction recursively. Finally define bi-infinite sequence $\omega \in \mathscr{C}^{\mathbb{Z}}$ by

$$
\omega_{\left[-l_{n}, l_{n}\right)}=F_{n} L_{n}
$$

and put $\Omega=\overline{\operatorname{Orb}(\omega, \sigma)}$.

TOME $140-2012-\mathrm{N}^{\mathrm{O}} 3$ 
Lemma 8.1. - Assume that

$$
\sum_{n=1}^{\infty} \frac{1}{3^{k_{n}}}+\sum_{n=1}^{\infty} \frac{2+m_{n}}{3^{k_{n+1}}}=\theta<\frac{2}{3}
$$

Then there is a sequence of permutations $\phi_{n}: \mathscr{G}_{n} \rightarrow \mathscr{G}_{n}$ such that for every $n$ and every $w \in \mathscr{G}_{n}$ the following holds:

$$
\alpha_{n}(w)=\frac{1}{l_{n}}\left\{i: w[i] \neq \phi_{n}(w)[i]\right\} \geq \frac{2}{3}-\theta>0 .
$$

Proof. - Let $\psi$ be a cycle on the set $\left\{1,2, \ldots, 3^{k_{0}}\right\}$. For any permutation $\sigma \in \Pi\left(3^{k_{0}}\right)$ define

$$
\phi_{1}\left(B_{\sigma}^{(1)}\right)=B_{\psi \circ \sigma}^{(1)}
$$

Then $B_{\sigma}^{(1)}[i] \neq B_{\psi \circ \sigma}^{(1)}[i]$ for every $i$ such that $B_{k_{0}}[i]=\diamond$. But then

$$
\alpha_{1}(w)=\frac{1}{l_{1}} \#\left\{i: w[i] \neq \phi_{1}(w)[i]\right\}=\frac{3^{k_{0}}}{\frac{1}{2}\left(3^{k_{0}+1}-1\right)}>\frac{2}{3}
$$

Next assume that for every $j=1, \ldots, n$ we have already constructed a permutation $\phi_{j}: \mathscr{G}_{j} \rightarrow \mathscr{G}_{j}$ such that $\hat{\alpha}_{n}=\min _{w \in \mathscr{G}_{n}} \alpha_{n}(w)$ satisfies the inequality

$$
\begin{aligned}
\hat{\alpha}_{j} & \geq \frac{2}{3}-\sum_{i=1}^{j-1} \frac{1}{3^{k_{i}}}-\sum_{i=1}^{j-1} \frac{m_{i}+2}{3^{k_{i+1}}} \\
& \geq \frac{2}{3}-\theta .
\end{aligned}
$$

We are going to show how $\phi_{n+1}: \mathscr{G}_{n+1} \rightarrow \mathscr{G}_{n+1}$ can be constructed. Fix any function $\sigma:\left\{1, \ldots, t_{n+1} m_{n}\right\} \rightarrow\left\{1, \ldots, m_{n}\right\}$. By the definition

$$
B_{\sigma}^{(n+1)}=B_{n+1} \downarrow\left(L_{n}\right)^{u_{n}+1} B_{\sigma(1)}^{(n)} \cdots B_{\sigma\left(t_{n+1 m_{n}}\right)}^{(n)} F_{n}
$$

and thus if we put

$$
\phi_{n+1}\left(B_{\sigma}^{(n+1)}\right)=B_{n+1} \downarrow\left(L_{n}\right)^{u_{n}+1} \phi_{n}\left(B_{\sigma(1)}^{(n)}\right) \cdots \phi_{n}\left(B_{\sigma\left(t_{n+1 m_{n}}\right)}^{(n)}\right) F_{n}
$$


then $\phi_{n+1}$ is well defined on any element of $\mathscr{G}_{n+1}$, and it is also invertible, since $\phi_{n}$ is invertible. To complete the proof, it is enough to calculate that

$$
\begin{aligned}
\alpha_{n+1}\left(B_{\sigma}^{(n+1)}\right) & =\frac{1}{l_{n+1}} \#\left\{i: B_{\sigma}^{(n+1)}[i] \neq \phi_{n+1}\left(B_{\sigma}^{(n+1)}\right)[i]\right\} \\
& \geq \frac{1}{l_{n+1}} \sum_{j=1}^{t_{n+1} m_{n}} \#\left\{i: B_{\sigma(j)}^{(n)}[i] \neq \phi_{n}\left(B_{\sigma(j)}^{(n)}\right)[i]\right\} \\
& \geq \frac{1}{l_{n+1}} \sum_{j=1}^{t_{n+1} m_{n}} l_{n} \hat{\alpha}_{n}=t_{n+1} m_{n} \hat{\alpha}_{n} \frac{l_{n}}{l_{n+1}} \\
& \geq \hat{\alpha}_{n}\left(3^{k_{n+1}}-m_{n}-2\right)\left(\frac{1}{3^{k_{n+1}}}-\frac{1}{3^{p_{n+1}+1}}\right) \\
& \geq \hat{\alpha}_{n}-\frac{m_{n}+2}{3^{k_{n+1}}}-\frac{3^{k_{n+1}}}{3^{p_{n+1}+1}} \\
& \geq \hat{\alpha}_{n}-\frac{m_{n}+2}{3^{k_{n+1}}}-\frac{1}{3^{k_{n}}} \\
& \geq \frac{2}{3}-\sum_{i=1}^{n} \frac{1}{3^{k_{i}}}-\sum_{i=1}^{n} \frac{m_{i}+2}{3^{k_{i+1}}} .
\end{aligned}
$$

since obviously $\hat{\alpha}_{n+1}=\alpha_{n+1}\left(B_{\sigma}^{(n+1)}\right)$ for some $\sigma$.

THEOREM 8.2. - Let $\Omega$ be the extension of the Chacón flow constructed above and assume that

$$
2 \sum_{n=1}^{\infty} \frac{1}{3^{k_{n}}}+\frac{3}{2} \sum_{n=1}^{\infty} \frac{2+m_{n}}{3^{k_{n+1}}}<1 .
$$

Then $\Omega$ is minimal with positive topological entropy and has $(\mathscr{M}(1), \mathscr{M}(a))-1 / 2$-scrambled set for some $a>0$.

Proof. - The fact that $\Omega$ is minimal with positive topological entropy follows by [8, Corollary $1 \&$ Proposition 1], so it remains to construct an $(\mathscr{M}(1), \mathscr{M}(a))-1 / 2$-scrambled set.

First note that if we fix any $\sigma \in \Phi_{n}$ then as the suffix of $B_{\sigma}^{(n+1)}$ we will have the block

$$
B_{\sigma\left(t_{n+1} m_{n}-1\right)}^{(n)} B_{\sigma\left(t_{n+1} m_{n}\right)}^{(n)} * F_{n} .
$$

By the same argument, there is $\gamma \in \Phi_{n+1}$ such that $B_{\gamma\left(t_{n+2} m_{n+1}\right)-1}^{(n+1)}=B_{\sigma}^{(n+1)}$ and so the suffix of $B_{\gamma}^{(n+2)}$ has the form

$$
B_{\sigma\left(t_{n+1} m_{n}-1\right)}^{(n)} B_{\sigma\left(t_{n+1} m_{n}\right)}^{(n)} * F_{n} B_{\gamma\left(t_{n+2} m_{n+1}\right)}^{(n+1)} * F_{n+1} .
$$

This shows that for every sequence $\sigma_{n} \in \Phi_{n}$ there is a point $x \in \Omega$ such that

$$
x_{[0,+\infty)}=B_{\sigma_{1}}^{(2)} * F_{2} B_{\sigma_{2}}^{(3)} * F_{3} B_{\sigma_{3}}^{(4)} * F_{4} \cdots
$$

TOME $140-2012-\mathrm{N}^{\circ} 3$ 
Let $D$ be the set of all points that can be defined that way. Observe that each block of the form

$$
B_{\sigma_{1}}^{(2)} * F_{2} B_{\sigma_{2}}^{(3)} * F_{3} \cdots B_{\sigma_{n-1}}^{(n)} * F_{n}
$$

is a suffix of $B_{\gamma}^{(n+1)}$ for some $\gamma \in \Phi_{n}$ and the length of this suffix is shorter than $\frac{1}{2} l_{n+1}$. This shows that $D$ does not have isolated points. Obviously it is also closed, thus perfect.

Let $\theta$ be provided by Lemma 8.1. For any $l, s>0$ define a set $A_{l}^{s} \subset D \times D$ such that $(x, y) \in A_{l}^{s}$ if and only if there is $k>s$ such that

$$
\frac{1}{k} \#\left\{0 \leq i<k: x_{[i, i+l]}=y_{[i, i+l]}\right\}>\frac{s-1}{s} .
$$

We claim that this set is dense in $D \times D$ (it is clear that it is open in $D \times D$ ). Fix any open sets $U, V$ such that $U \cap D \neq \varnothing, V \cap D \neq \varnothing$ There are $n>0$ and sequences of permutations $\sigma_{1}, \ldots, \sigma_{n-1}, \eta_{1}, \ldots, \eta_{n-1}$ such that we have inclusion of cylinder sets $C_{\Omega}[u] \subset U, C_{\Omega}[v] \subset V$ where

$$
\begin{aligned}
& u=B_{\sigma_{1}}^{(2)} * F_{2} B_{\sigma_{2}}^{(3)} * F_{3} \cdots B_{\sigma_{n-1}}^{(n)} * F_{n} \\
& v=B_{\eta_{1}}^{(2)} * F_{2} B_{\eta_{2}}^{(3)} * F_{3} \cdots B_{\eta_{n-1}}^{(n)} * F_{n}
\end{aligned}
$$

Note that $|u|=|v|=n-1+2 \sum_{j=2}^{n} l_{n}$ and $3^{p_{n}} / 4 \leq l_{n} \leq 3^{p_{n}+1} / 2$. Then for $n$ sufficiently large, in particular $n>l$ we get

$$
\begin{aligned}
\frac{|u|+l+1}{l_{n+1}} & \leq \frac{2 n+2 \sum_{j=2}^{n} l_{j}}{l_{n+1}} \\
& \leq \frac{2 n}{3^{n}}+\frac{6 \sum_{j=2}^{n} 3^{p_{j}} / 2}{3^{p_{n+1}} / 4} \\
& \leq \frac{2 n}{3^{n}}+\frac{12 n}{3^{k_{n+1}}} \leq \frac{14 n}{3^{n}} \longrightarrow 0 .
\end{aligned}
$$

Now, consider $x, y \in D$ with prefixes $u * B_{\gamma}^{(n+1)}, v * B_{\gamma}^{(n+1)}$ respectively, where $B_{\gamma}^{(n+1)}$ is a fixed word in $\mathscr{G}_{n+1}$ (such words $x, y$ exist by the definition of $D$ ). As an immediate consequence we see that for sufficiently large $n$ and $k=$ $|u|+l_{n+1}+1$ we have

$$
\frac{1}{k} \#\left\{0 \leq i<k: x_{[i, i+l]}=y_{[i, i+l]}\right\} \geq 1-\frac{|u|+l+1}{l_{n+1}}>\frac{s-1}{s} .
$$

Indeed, each set $A_{l}^{s} \subset D \times D$ is open and dense.

Next, observe that by the assumptions

$$
\frac{3}{2} \sum_{n=1}^{\infty} \frac{1}{3^{k_{n}}}+\frac{3}{2} \sum_{n=1}^{\infty} \frac{2+m_{n}}{3^{k_{n+1}}}<1 .
$$

BULLETIN DE LA SOCIÉtÉ MATHÉMATIQUE DE FRANCE 
and so we are allowed to use Lemma 8.1. Consider the following number

$$
c_{x y}^{k}=\frac{1}{k} \#\left\{0 \leq i<k: x_{i} \neq y_{i}\right\}
$$

for some fixed words $x, y$ with prefixes $u, v$ respectively. Assume even more, that as before the prefix of $x$ is $u * B_{\gamma}^{(n+1)}$ while in the case of $y$ we have the prefix $u * \phi_{n+1}\left(B_{\gamma}^{(n+1)}\right)$, where the function $\phi_{n+1}$ is a permutation provided by Lemma 8.1. For $k=|u|+1+l_{n+1}$, putting $\delta=\frac{2}{3}-\theta>0$, we can calculate that

$$
\begin{aligned}
c_{x y}^{k} & \geq \frac{\delta l_{n+1}}{l_{n+1}+|u|+1} \geq \delta-\frac{2|u|}{l_{n+1}} \\
& \geq \delta-\frac{4 n l_{n}}{l_{n+1}} \geq \delta-\frac{4 n l_{n}}{\frac{1}{2} 3^{k_{n}} l_{n}} \\
& \geq \delta-\frac{8 n}{3^{n}} \longrightarrow \delta .
\end{aligned}
$$

Now, consider the set $C_{l} \subset D \times D$ consisting of pairs $(x, y)$ such that for some $k>l$ the following inequality is satisfied

$$
\frac{1}{k} \#\left\{0 \leq i<k: x_{i} \neq y_{i}\right\}>\varepsilon
$$

with $\varepsilon=\frac{1}{2}\left(\frac{2}{3}-\theta\right)$. Simply by the definition $C_{l}$ is open and by previous calculations it is also a dense subset of $D \times D$ for every $l>0$. As an immediate consequence we obtain that the set

$$
R=\bigcap_{l, s=1}^{\infty}\left(A_{l}^{s} \cap C_{l}\right)
$$

is a residual subset of $D \times D$ and for every $(x, y) \in R$ and every $t>0$ we have

$$
\Phi_{x y}^{*}(t)=1 \quad \text { and } \quad \Phi_{x y}(1) \leq 1-\varepsilon .
$$

In particular it means that

$$
(x, y) \in \mathscr{M}(1)^{+}(\Delta, \sigma \times \sigma) \cap \mathscr{M}(a)\left(\Omega \times \Omega \backslash \Delta_{1 / 2}, \sigma \times \sigma\right)
$$

where $a=\varepsilon / 2$. It remains to apply Theorem 7.5 and the proof is completed.

\section{Embedded $k$-blocks and $(\mathscr{M}(1), \mathscr{M}(1))$-chaos}

Almost 1-1 extensions are one of the most prominent techniques of construction of minimal systems with prescribed properties. One starts with a system with well studied dynamics and then extend it to another one, by splitting some of fibres in a way that a much richer dynamics is obtained. Unfortunately, as we demonstrated in Section $4,(\mathscr{M}(1), \mathscr{M}(1))$-chaotic system cannot arise that way.

TOME $140-2012-\mathrm{N}^{\mathrm{O}} 3$ 
According to the best knowledge of the author there is only one general technique of the construction of DC1 minimal systems known in the literature, which originated from [22] and was later used by various authors. However, by similar reasons as in Section 3, topological entropy of systems constructed by this scheme must be zero. Here we will combine the technique of embedded $k$-blocks (inspired by the construction from [8]) with some ideas of [22]. That way a minimal weakly mixing and $(\mathscr{M}(1), \mathscr{M}(1))$-chaotic system with positive topological entropy will be constructed. A question whether such a system exists was stated explicitly in [4, Problem (ii)].

Notation used in this section will be very similar to that in Section 8. Fix any $k_{0}>2$ and let $\mathscr{G}=\left\{1,2, \ldots, k_{0}\right\}$. Let

$$
\mathscr{G}_{1}=\left\{\sigma(1) \sigma(2) \cdots \sigma\left(k_{0}\right): \sigma \in \Pi\left(k_{0}\right)\right\} \cup\left\{a^{k_{0}+1}: a=1, \ldots, k_{0}\right\} .
$$

We put $m_{1}=\# \mathscr{G}_{1}=k_{0} !+k_{0}$. Let $t_{n}$ be an increasing sequence (it will be specified more precisely later). For every $n$ (under assumption that the number $m_{n}$ is defined), let $\Phi_{n}$ denote the set of maps $\phi:\left\{1, \ldots, t_{n+1} m_{n}\right\} \rightarrow$ $\left\{1, \ldots, m_{n}\right\}$ such that each fiber has the same number of elements, that is $\# \phi^{-1}(t)=t_{n+1}$ for every $t \in\left\{1, \ldots, m_{n}\right\}$.

The family of words $\mathscr{C}_{n}$ is defined recursively in the following way. Assume that $\mathscr{G}_{n}$ is defined and enumerate its elements $\mathscr{G}_{n}=\left\{B_{1}^{(n)}, \ldots, B_{m_{n}}^{(n)}\right\}$ where $m_{n}=\# \mathscr{G}_{n}$. We put

$$
\mathscr{G}_{n+1}=\left\{B_{\sigma(1)}^{(n)} B_{\sigma(2)}^{(n)} \cdots B_{\sigma\left(m_{n} t_{n+1}\right)}^{(n)} S: \sigma \in \Phi_{n}, S \in \mathscr{G}_{n}\right\} .
$$

We assume additionally that $B_{1}^{(n)}$ is a prefix of $B_{1}^{(n+1)}$, where $B_{1}^{(n+1)}$ is the first word in $\mathscr{G}_{n+1}$ after enumeration of its elements. Note that each $w \in \mathscr{G}_{n+1}$ can be uniquely decomposed into elements of $\mathscr{G}_{1}$ and then (since the number of elements of $\mathscr{G}_{i}$ used to construct an element of $\mathscr{G}_{i+1}$ is always the same), decomposition of $w$ into elements of $\mathscr{G}_{n}$ is also unique.

Finally we define $x$ to be the unique infinite sequence beginning with $B_{1}^{(n)}$ for every $n$, and let $X=\overline{\operatorname{Orb}^{+}(x, \sigma)}$ be the generated subshift.

Lemma 9.1. - For every $n>0$ there is a permutation $\phi_{n}: \mathscr{G}_{n} \rightarrow \mathscr{G}_{n}$ such that $\left|\phi_{n}(w)\right|=|w|$ and $\left\{0 \leq i<|w|: w[i]=\phi_{n}(w)[i]\right\}=\varnothing$ for every $w \in \mathscr{C}_{n}$.

Proof. - When $n=1$ it is easy. Namely, we fix a cycle $\psi \in \Pi\left(k_{0}\right)$ and $\phi_{1}\left(w_{1} \cdots w_{k}\right)=\psi\left(w_{1}\right) \cdots \psi\left(w_{k}\right)$ where $k=k_{0}$ or $k_{0}+1$ depending on the choice of word in $\mathscr{C}_{1}$. 
Next, if $w \in \mathscr{G}_{n+1}$ then $w=B_{\sigma(1)}^{(n)} B_{\sigma(2)}^{(n)} \cdots B_{\sigma\left(m_{n} t_{n+1}\right)}^{(n)} S$ for some $\sigma \in$ $\Phi_{n}, S \in \mathscr{G}_{n}$, and so we can define

$$
\begin{aligned}
\phi_{n+1}(w) & =\phi_{n}\left(B_{\sigma(1)}^{(n)}\right) \phi_{n}\left(B_{\sigma(2)}^{(n)}\right) \cdots \phi_{n}\left(B_{\sigma\left(m_{n} t_{n+1}\right)}^{(n)}\right) \phi_{n}(S) \\
& =B_{\sigma^{\prime}(1)}^{(n)} B_{\sigma^{\prime}(2)}^{(n)} \cdots B_{\sigma^{\prime}\left(m_{n} t_{n+1}\right)}^{(n)} S^{\prime}
\end{aligned}
$$

where $\sigma^{\prime} \in \Phi_{n}, S^{\prime} \in \mathscr{C}_{n}$ are uniquely determined by $\phi_{n}$. Obviously $\phi_{n+1}$ defined that way is invertible since $\phi_{n}$ is. The remaining properties of $\phi_{n+1}$ easily follow by induction.

Lemma 9.2. - For every $n>0$ and every $A, B \in \mathscr{G}_{n}$ we have ||$A|-| B|| \leq 1$ and $|A| /|B|<2$. Furthermore, for every $n=1,2, \ldots$ we can find $A, B \in \mathscr{G}_{n}$ so that $|A|-|B|=1$.

Proof. - The proof easily follows by induction.

TheOREM 9.3. - There is a sequence $t_{n}$ such that the shift $X$ defined above has the following properties:

1. is minimal,

2. is weakly mixing,

3. has distributionally 1/2-scrambled set (in particular is $(\mathscr{M}(1), \mathscr{M}(1))$-chaotic),

4. $h_{\text {top }}(X)>0$.

Proof. - For the proof of conditions (1)-(3) it is enough to assume that $t_{n}$ is increasing. We will later specify how fast $t_{n}$ should grow to ensure also (4). The proof of (1) is standard (see the proof of Lemma 3.3) thus left to the reader.

Now we are going to prove (2). For $n>2$, clearly $t_{n} \geq 3$ and so for any $S \in \mathscr{A}_{n}$ the word $B_{1}^{(n)} S B_{1}^{(n)}$ must appear somewhere in $x$. If we fix any neighborhood $U$ of $x$ then for sufficiently large $n$ we have $U \supset C_{X}\left[B_{1}^{(n)}\right]$ and so by Lemma 9.2 we immediately obtain that $k, k+1 \in N(U, U, \sigma)$ for some $k>0$, thus (2) follows by Lemma 3.8.

The proof of (3) is a little more complicated. Let $D \subset X$ consists of points that can be obtained as the limit of a sequence of words $w_{n} \in \mathscr{G}_{n}$. Any word from $\mathscr{G}_{n}$ is a prefix of at least two words in $\mathscr{G}_{n+1}$, so $D$ does not have isolated points. It is also easy to verify that it is compact. Namely, for any sequence $\left\{x_{n}\right\}_{n=1}^{\infty}$ we select an infinite subsequence $\left\{y_{n}\right\}_{n=1}^{\infty}$ having the same word $w_{1} \in$ $\mathscr{G}_{1}$ as a prefix of all its elements. Next we take a subsequence of $\left\{y_{n}\right\}_{n=1}^{\infty}$ having the same word from $w_{2} \in \mathscr{G}_{2}$ as a prefix and so on (obviously $w_{1}$ must be a prefix of $w_{2}$ ). That way a convergent subsequence is produced and its limit $z$ has all words $w_{n} \in \mathscr{G}_{n}$ selected above as its prefixes, thus $z \in D$. Indeed, $D$ is a perfect set. 
Next, we claim that $\mathrm{DC}_{1 / 2}(\sigma) \cap(D \times D)$ is residual in $D \times D$. First, for any $l, s>0$ define a set $A_{l}^{s} \subset D \times D$ such that $(x, y) \in A_{l}^{s}$ if and only if there is $k>s$ such that

$$
\frac{1}{k} \#\left\{0 \leq i<k: x_{[i, i+l]}=y_{[i, i+l]}\right\}>\frac{s-1}{s} .
$$

Note that every set $A_{l}^{s}$ is open in $D \times D$ and if $(x, y) \in \bigcap_{l, s=1}^{\infty} A_{l}^{s}$ then $\Phi_{x y}^{*}(t)=1$ for every parameter $t>0$. We are going to show that sets $A_{l}^{s}$ are dense. Fix any open sets $U, V$ intersecting $D$ and let $u, v \in \mathscr{Q}^{*}$ be such that $C_{X}[u] \subset U$, $C_{X}[v] \subset V$. There are $n>0$ and $\tau, \xi$ such that $u$ is a prefix of $B_{\tau}^{(n)}, v$ is a prefix of $B_{\xi}^{(n)}$ and $m_{n-1}>\max \{6 s, l\}$. We can also assume that $\left|B_{\tau}^{(n)}\right|=$ $\left|B_{\xi}^{(n)}\right|$. To simplify further notation assume that $\tau=1$ and $\xi=m_{n}$. If we put $\phi(i)=1+\left(i-1\left(\bmod m_{n}\right)\right)$ for $i=1, \ldots, t_{n+1} m_{n}$ then $\phi \in \Phi_{n}$. Define $\psi$ to be equal to $\phi$ with the only exception that $\psi(1)=\phi\left(t_{n+1} m_{n}\right)=m_{n}$, $\psi\left(t_{n+1} m_{n}\right)=\phi(1)=1$. Note that if $B \in \mathscr{G}_{n}$ then $|B| \geq m_{n-1}>l$. But now, if we consider words $B_{\psi}=B_{\psi(1)}^{(n)} \cdots B_{\psi\left(t_{n+1} m_{n}\right)}^{(n)}$ and $B_{\phi}=B_{\phi(1)}^{(n)} \cdots B_{\phi\left(t_{n+1} m_{n}\right)}^{(n)}$ (and put $k=\left|B_{\psi}\right|$ ) then $u, v$ are their respective prefixes, and additionally

$$
\begin{aligned}
\frac{1}{k}\left\{0 \leq i<k:\left(B_{\psi}\right)_{[i, i+l]}=\left(B_{\phi}\right)_{[i, i+l]}\right\} & \geq \frac{1}{k}\left(k-3 \max _{w \in \mathscr{Q}_{n}}|w|\right) \\
& \geq 1-\frac{3}{m_{n}} \geq \frac{s-1}{s}
\end{aligned}
$$

since by Lemma 9.2 we have $\left|B_{i}^{(n)}\right| / k<2 / t_{n+1} m_{n}<1 / m_{n}$ for every $i=$ $1, \ldots, m_{n}$. But obviously, there are $x, y \in D$ such that $B_{\psi}$ is a prefix of $x$ and $B_{\phi}$ is a prefix of $y$, which immediately implies that $A_{l}^{s}$ is dense in $D \times D$.

Next, for any $l, s>0$ define a set $R_{l}^{s} \subset D \times D$ such that $(x, y) \in R_{l}^{s}$ if and only if there is $k>l$ such that

$$
\frac{1}{k} \#\{0 \leq i<k: x[i] \neq y[i]\}>\frac{s-1}{s} .
$$

Again, it is obvious that $R_{l}^{s}$ is open and if $(x, y) \in \bigcap_{l, s=1}^{\infty} R_{l}^{s}$ then $\Phi_{x y}(1 / 2)=0$. But, similarly to the case of $A_{l}^{s}$ we can prove that $R_{l}^{s}$ is dense. More precisely, let all objects, including $\psi$, be as defined above (for the case of $A_{l}^{s}$ ). Let $\eta \in \Phi_{n}$ be the map such that

$$
B_{\eta}=\phi_{n}\left(B_{\psi(1)}^{(n)}\right) \phi_{n}\left(B_{\psi(2)}^{(n)}\right) \cdots \phi_{n}\left(B_{\psi\left(m_{n} t_{n+1}\right)}^{(n)}\right)=B_{\eta(1)}^{(n)} B_{\eta(2)}^{(n)} \cdots B_{\eta\left(m_{n} t_{n+1}\right)}^{(n)}
$$

where the map $\phi_{n}$ is provided by Lemma 9.1. If $\eta(1)=m_{n}$ then we put $\phi=\eta$ and $j=2$. Otherwise we find the first position $j$ such that $\eta(j)=m_{n}$ and we define $\phi$ to be equal $\eta$ with the only exception that $\phi(1)=\eta(j)=m_{n}$ and $\phi(j)=\eta(1)$. Then $\phi \in \Phi_{n}$ and additionally, for at least $t_{n+1} m_{n}-2$ indices $p$ we have $\left|B_{\psi(p)}^{(n)}\right|=\left|B_{\phi(p)}^{(n)}\right|$ and $B_{\psi(p)}^{(n)}[i] \neq B_{\phi(p)}^{(n)}[i]$ for every $0 \leq i<\left|B_{\psi(p)}^{(n)}\right|$. Similarly to the case of $A_{l}^{s}$ we have $C_{X}\left[B_{\psi(1)}\right] \subset U, C_{X}\left[B_{\phi(1)}\right] \subset V$. We can 
also repeat all previous calculations (extending $B_{\psi}, B_{\phi}$ to $x$ and $y$ respectively), obtaining

$$
\begin{aligned}
\frac{1}{k} \#\left\{0 \leq i<k: B_{\psi}[i] \neq B_{\phi}[i]\right\} & \geq \frac{1}{k}\left(k-\left|B_{\psi(1)}^{(n)}\right|-\left|B_{\psi(j)}^{(n)}\right|\right) \\
& \geq 1-\frac{2}{m_{n}} \geq \frac{s-1}{s} .
\end{aligned}
$$

Since sets $A_{l}^{s}, R_{s}^{l}$ are open and dense in $D \times D$, the set $\bigcap_{l, s=1}^{\infty}\left(A_{l}^{s} \cap R_{l}^{s}\right)$ is residual in $D \times D$. But we also have that

$$
\bigcap_{l, s=1}^{\infty}\left(A_{l}^{s} \cap R_{l}^{s}\right) \subset \mathrm{DC}_{1 / 2}(\sigma) \cap(D \times D) .
$$

which by Theorem 7.5 implies that $D$ contains a Cantor set $C$ such that $C \times C \subset$ $\mathrm{DC}_{1 / 2}(\sigma) \cup \Delta$. In other words $C \subset X$ is distributionally $1 / 2$-scrambled set.

The proof of (4) is a direct calculation, which uses to some extent ideas of [8]. Denote by $s_{n}$ the longest word in $\mathscr{G}_{n}$ and try to estimate the number $c_{n}$ of words of length $s_{n}$. Obviously $m_{n} \leq c_{n}$, so $h_{\text {top }}(X) \geq \lim \sup _{n \rightarrow \infty} \frac{\log \left(m_{n}\right)}{s_{n}}$. Denote $\tau_{n}=\log \left(m_{n}\right) / s_{n}$. We also assume that $t_{n}$ satisfies the following two conditions:

$$
\left(1+\frac{1}{t_{n}}\right) \leq 2^{\frac{1}{2^{n}}}, \quad t_{n} \geq 2 \beta^{n+1} \quad \text { where } \beta=2 k_{0}+2 .
$$

Before we proceed with calculations, make additional two observations:

$$
m_{n+1} \geq \frac{\left(m_{n} t_{n+1}\right) !}{\left(t_{n+1} !\right)^{m_{n}}}, \quad s_{n+1} \leq\left(m_{n} t_{n+1}+1\right) s_{n} .
$$

Additionally, it is known by Stirling's formula that $n \log (n)-n \leq \log (n$ !) $\leq$ $n \log (n)$ and also $s_{n} \geq t_{n}$. Then we can calculate that

$$
\begin{aligned}
& \frac{\log \left(m_{n+1}\right)}{s_{n+1}} \geq \frac{m_{n} t_{n+1}\left(\log \left(m_{n} t_{n+1}\right)-1-\log \left(t_{n+1}\right)\right)}{\left(m_{n} t_{n+1}+1\right) s_{n}} \\
& \geq \frac{1}{1+\frac{1}{m_{n} t_{n+1}}} \frac{\log \left(m_{n}\right)}{s_{n}}-\frac{1}{t_{n}} \\
& \geq 2^{-\frac{1}{2^{n+1}}} \tau_{n}-\frac{1}{\beta^{n+1}} \geq \prod_{j=1}^{n} 2^{-\frac{1}{2^{j+1}}} \tau_{1}-\sum_{j=1}^{n} \frac{1}{\beta^{j+1}} \\
& \geq 2^{-1 / 2} \tau_{1}-\frac{1}{\beta(\beta-1)} \geq \frac{\log \left(k_{0}\right)-1}{\beta}>0 .
\end{aligned}
$$

We have just proved that $h_{\mathrm{top}}(X) \geq \frac{\log \left(k_{0}\right)-1}{\beta}>0$ and thus the proof is completed. 
Recall that a closed set $J \subset X \times Y$ is a joining of $(X, f)$ and $(Y, g)$ if it is invariant (for the product map $f \times g$ ) and its projections on first and second coordinate are $X$ and $Y$ respectively. If each joining is equal to $X \times Y$ then we say that $(X, f)$ and $(Y, g)$ are disjoint.

The following example shows that assumptions of Corollary 4.2 are essential (in particular, constructed example cannot be almost 1-1 extension of odometer).

EXAMPLE 9.4. - Every odometer has an extension which is minimal and has a distributionally $\varepsilon$-scrambled set (so it is $(\mathscr{M}(1), \mathscr{M}(1)$ )-chaotic).

Proof. - Let $(X, \sigma)$ be the minimal system from Theorem 9.3 and let $(Y, f)$ be an odometer. Consider $F=\sigma \times f$ and $Z=X \times Y$ endowed with the product topology and the standard maximum metric $\rho$ (maximum of distance on coordinates). Obviously $(Z, F)$ is an extension of $(Y, f)$ and it is also known that minimal distal systems are disjoint from weakly mixing systems (see [10, Theorem 2.3]). So $Z$ is the only joining of $(X, \sigma),(Y, f)$ and thus $F$ must be minimal on $Z$. If we take a distributionally $\varepsilon$-scrambled set $S \subset X$ then by the definition of metric in $Z$ we immediately see that $S \times\{y\}$ is also distributionally $\varepsilon$-scrambled for any fixed $y \in Y$ which ends the proof.

\section{Acknowledgements}

The author is grateful to teh unknown referee of this paper for his/her careful reading. The author express his thanks to F. Blanchard and T. Downarowicz for numerous remarks on techniques of construction of minimal systems with positive topological entropy, in particular for pointing out [32], and also to $\mathrm{S}$. Williams for providing the author with a copy of that interesting article. The author would like to thank J. Kwiatkowski and D. Kwietniak for pointing out papers of Grillenberger (especially [13]) and useful comments on results contained therein.

This work was supported by the Polish Ministry of Science and Higher Education from sources for science in the years 2010-2011, grant no. IP2010 029570.

A part of the results included in the paper was obtained when the author was visiting Universidad de Murcia, supported by Marie Curie Intra-European Fellowship for Career Development (grant agreement no. 219212), MICINN (Ministerio de Ciencia e Innovación) and FEDER (Fondo Europeo de Desarrollo Regional), grant MTM2008-03679/MTM.

The financial support of these institutions is hereby gratefully acknowledged. 


\section{BIBLIOGRAPHY}

[1] R. L. Adler, A. G. Konheim \& M. H. MCAndrew - "Topological entropy", Trans. Amer. Math. Soc. 114 (1965), p. 309-319.

[2] E. AKIN - "Lectures on Cantor and Mycielski sets for dynamical systems", in Chapel Hill Ergodic Theory Workshops, Contemp. Math., vol. 356, Amer. Math. Soc., 2004, p. 21-79.

[3] F. Balibrea, B. Schweizer, A. Sklar \& J. Smítal - "Generalized specification property and distributional chaos", Internat. J. Bifur. Chaos Appl. Sci. Engrg. 13 (2003), p. 1683-1694, Dynamical systems and functional equations (Murcia, 2000).

[4] F. Balibrea \& J. Smítal - "Strong distributional chaos and minimal sets", Topology Appl. 156 (2009), p. 1673-1678.

[5] F. Balibrea, J. Smítal \& M. Štefánková - "The three versions of distributional chaos", Chaos Solitons Fractals 23 (2005), p. 1581-1583.

[6] J. BANKS - "Regular periodic decompositions for topologically transitive maps", Ergodic Theory Dynam. Systems 17 (1997), p. 505-529.

[7] F. Blanchard, B. Host \& S. Ruette - "Asymptotic pairs in positiveentropy systems", Ergodic Theory Dynam. Systems 22 (2002), p. 671-686.

[8] F. Blanchard \& J. KwiatKowski - "Minimal self-joinings and positive topological entropy. II", Studia Math. 128 (1998), p. 121-133.

[9] T. Downarowicz - "Survey of odometers and Toeplitz flows", in Algebraic and topological dynamics, Contemp. Math., vol. 385, Amer. Math. Soc., 2005, p. 7-37.

[10] H. FurstenberG - "Disjointness in ergodic theory, minimal sets, and a problem in Diophantine approximation", Math. Systems Theory 1 (1967), p. $1-49$.

[11] , Recurrence in ergodic theory and combinatorial number theory, Princeton Univ. Press, 1981.

[12] C. Grillenberger - "Constructions of strictly ergodic systems. I. Given entropy", Z. Wahrscheinlichkeitstheorie und Verw. Gebiete 25 (1972/73), p. 323-334.

[13] _ "Constructions of strictly ergodic systems. II. K-Systems", $Z$. Wahrscheinlichkeitstheorie und Verw. Gebiete 25 (1972/73), p. 335-342.

[14] C. Grillenberger \& P. Shields - "Construction of strictly ergodic systems. III. Bernoulli systems", Z. Wahrscheinlichkeitstheorie und Verw. Gebiete 33 (1975/76), p. 215-217.

[15] L. HE \& C. LIU - "Invariant measures and uniform positive entropy property for inverse limits", Appl. Math. J. Chinese Univ. Ser. B 14 (1999), p. 265-272, A Chinese summary appears in Gaoxiao Yingyong Shuxue Xuebao Ser. A 14 (1999), no. 3, 367. 
[16] W. HuAng \& X. YE - "Devaney's chaos and 2-scattering imply Li-Yorke's chaos", Topology 117 (2002), p. 259-272.

[17] W. Huang, S. Shao \& X. YE - "Mixing via sequence entropy", in $A l$ gebraic and topological dynamics, Contemp. Math., vol. 385, Amer. Math. Soc., 2005, p. 101-122.

[18] W. HuAng \& X. YE - "A local variational relation and applications", Israel J. Math. 151 (2006), p. 237-279.

[19] D. KERR \& H. Li - "Independence in topological and $C^{*}$-dynamics", Math. Ann. 338 (2007), p. 869-926.

[20] P. KuRKA - Topological and symbolic dynamics, Cours Spécialisés, vol. 11, Soc. Math. France, 2003.

[21] T. Y. Li \& J. A. Yorke - "Period three implies chaos", Amer. Math. Monthly 82 (1975), p. 985-992.

[22] G. LiAo \& L. WANG - "Almost periodicity and distributional chaos", in Foundations of computational mathematics (Hong Kong, 2000), World Sci. Publ., River Edge, NJ, 2002, p. 189-210.

[23] P. Oprocha - "Distributional chaos revisited", Trans. Amer. Math. Soc. 361 (2009), p. 4901-4925.

[24] _ "Weak mixing and product recurrence", Ann. Inst. Fourier 60 (2010), p. 1233-1257.

[25] P. Oprocha \& M. Štefánková - "Specification property and distributional chaos almost everywhere", Proc. Amer. Math. Soc. 136 (2008), p. 3931-3940.

[26] P. Oprocha \& P. WilczyŃski - "Distributional chaos via semiconjugacy", Nonlinearity 20 (2007), p. 2661-2679.

[27] R. PikuŁA - "On some notions of chaos in dimension zero", Colloq. Math. 107 (2007), p. 167-177.

[28] B. Schweizer, A. Sklar \& J. Smítal - "Distributional (and other) chaos and its measurement", Real Anal. Exchange 26 (2000/01), p. 495524 .

[29] B. Schweizer \& J. Smítal - "Measures of chaos and a spectral decomposition of dynamical systems on the interval", Trans. Amer. Math. Soc. 344 (1994), p. 737-754.

[30] A. Sklar \& J. Smítal - "Distributional chaos on compact metric spaces via specification properties", J. Math. Anal. Appl. 241 (2000), p. 181-188.

[31] F. TAN \& J. XIONG - "Chaos via Furstenberg family couple", Topology Appl. 156 (2009), p. 525-532.

[32] S. Williams - "Toeplitz minimal flows which are not uniquely ergodic", Z. Wahrsch. Verw. Gebiete 67 (1984), p. 95-107. 